\title{
Two Unknown Summation Formulae involving Contiguous relation
}

\author{
Intazar Husain ${ }^{1}$, Chaudhary Wali Mohd ${ }^{1}$. And Salahuddin ${ }^{2}$ \\ 1. Department of Applied Sciences \& Humanities \\ Jamia Millia Islamia, New Delhi, India \\ 2. . P.D.M university, Bahadurgarh, Haryana, India
}

\begin{abstract}
In this paper we have developed two summation formulae which are unknown. These formulae are developed with the help of contiguous relation and the formulae from Salahuddin et al.
\end{abstract}

Key Words: Special functions, Contiguous relation, Summation formulae, Salahuddin et al.

2010 MSC NO: 33C05, 33C20, 33C45, 33C60

\section{INTRODUCTION AND RESULTS REQUIRED}

Special functions are very useful according to their scope. So many problems related to the various areas of Science, Mathematical Analysis, Applied Mathematics can be solved by various types of special functions.

The discovery of a hypergeometric function has provided an intrinsic stimulation in the world of mathematics. It has also motivated the development of several domains such as complex functions, Riemann surfaces, differential equations, difference equations, arithmetic theory and so forth.

Summation formulae for hypergeometric function has an important role in applied mathematics. Prudnikov et al [2, p.414] derived the following seven summation formulae

$$
\begin{aligned}
& { }_{2} F_{1}\left[\begin{array}{rrr}
a, & -a ; & \frac{1}{2} \\
c & ; & 2
\end{array}\right] \\
& =\frac{\sqrt{\pi} \Gamma(c)}{2^{c}}\left[\frac{1}{\Gamma\left(\frac{c+a+1}{2}\right) \Gamma\left(\frac{c-a}{2}\right)}+\frac{1}{\Gamma\left(\frac{c+a}{2}\right) \Gamma\left(\frac{c-a+1}{2}\right)}\right]
\end{aligned}
$$

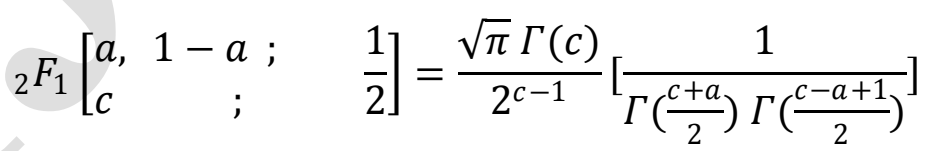

$$
\begin{aligned}
& { }_{2} F_{1}\left[\begin{array}{lrr}
a, & 2-a ; & \frac{1}{2} \\
c & ; & {[}
\end{array}\right] \\
& =\frac{\sqrt{\pi} \Gamma(c)}{(a-1) 2^{c-2}}\left[\frac{1}{\Gamma\left(\frac{c+a-2}{2}\right) \Gamma\left(\frac{c-a+1}{2}\right)}\right. \\
& \left.-\frac{1}{\Gamma\left(\frac{c+a-1}{2}\right) \Gamma\left(\frac{c-a}{2}\right)}\right]
\end{aligned}
$$




$$
\begin{aligned}
& { }_{2} F_{1}\left[\begin{array}{ccc}
a, & 3-a ; & \frac{1}{2} \\
c & ; &
\end{array}\right] \\
& =\frac{\sqrt{\pi} \Gamma(c)}{(a-1)(a-2) 2^{c-3}}\left[\frac{(c-2)}{\Gamma\left(\frac{c+a-2}{2}\right) \Gamma\left(\frac{c-a+1}{2}\right)}\right. \\
& \left.-\frac{2}{\Gamma\left(\frac{c+a-3}{2}\right) \Gamma\left(\frac{c-a}{2}\right)}\right]
\end{aligned}
$$

$$
\begin{aligned}
& { }_{2} F_{1}\left[\begin{array}{lrr}
a, & 4-a ; & \frac{1}{2} \\
c & ; &
\end{array}\right] \\
& =\frac{\sqrt{\pi} \Gamma(c)}{(1-a)(2-a)(3-a) 2^{c-4}}\left[\frac{(a-2 c+3)}{\Gamma\left(\frac{c+a-4}{2}\right) \Gamma\left(\frac{c-a+1}{2}\right)}\right. \\
& \left.+\frac{(a+2 c-7)}{\Gamma\left(\frac{c+a-3}{2}\right) \Gamma\left(\frac{c-a}{2}\right)}\right]
\end{aligned}
$$$$
{ }_{2} F_{1}\left[\begin{array}{lrr}
a, & 5-a ; & \frac{1}{2} \\
c & ; & 2
\end{array}\right]
$$$$
=\frac{\sqrt{\pi} \Gamma(c)}{2^{c-5}\left\{\prod_{\gamma=1}^{4}(\gamma-a)\right\}}\left[\frac{\{2(c-2)(c-4)-(a-1)(a-4)\}}{\Gamma\left(\frac{c-a+1}{2}\right) \Gamma\left(\frac{c+a-4}{2}\right)}\right.
$$$$
\left.+\frac{(12-4 c)}{\Gamma\left(\frac{c-a}{2}\right) \Gamma(\hat{r} a c c+a-52)}\right]
$$$$
{ }_{2} F_{1}\left[\begin{array}{ccc}
a, & 6-a ; & \frac{1}{2} \\
c & ; & =
\end{array}=\right.
$$

$$
\begin{gathered}
=\frac{\sqrt{\pi} \Gamma(c)}{2^{c-6}\left\{\prod_{\delta=1}^{5}(\delta-a)\right\}}\left[\frac{\left(4 c^{2}+2 a c-a^{2}-a-34 c+62\right)}{\Gamma\left(\frac{c-a}{2}\right) \Gamma\left(\frac{c+a-5}{2}\right)}\right. \\
\left.-\frac{\left(4 c^{2}-2 a c-a^{2}+13 a-22 c+20\right)}{\Gamma\left(\frac{c-a+1}{2}\right) \Gamma\left(\frac{c+a-6}{2}\right)}\right]
\end{gathered}
$$

The contiguous relation is defined as Abramowitz et al[1,p.558]

$$
\begin{aligned}
& \text { b }{ }_{2} F_{1}\left[\begin{array}{ccc}
a, & b+1 ; & z \\
c & ;
\end{array}\right] \\
& =(b-c+1) \quad{ }_{2} F_{1}\left[\begin{array}{lll}
a & b ; & z \\
c & ;
\end{array}\right]+(c \\
& \text {-1) }{ }_{2} F_{1}\left[\begin{array}{lll}
a, b ; & z \\
c-1 ; &
\end{array}\right]
\end{aligned}
$$

Salahuddin et al[2,4,5,6,7,8,9] derived the following summation formulae

$$
\begin{aligned}
& { }_{2} F_{1}\left[\begin{array}{ccc}
a & 7-a ; & \frac{1}{2} \\
c & ; &
\end{array}\right]= \\
& =\frac{\sqrt{\pi} \Gamma(c)}{2^{c-7}\left\{\prod_{\zeta=1}^{6}(\varsigma-a)\right\}}\left[\frac { 1 } { \Gamma ( \frac { c - a + 1 } { 2 } ) \Gamma ( \frac { c + a - 6 } { 2 } ) } \left(-3 a^{2} c+12 a^{2}+21 a c-84 a+4 c^{3}-48 c^{2}+158 c\right.\right. \\
& -120)+
\end{aligned}
$$




$$
\begin{aligned}
& \left.+\frac{1}{\Gamma\left(\frac{c-a}{2}\right) \Gamma\left(\frac{c+a-7}{2}\right)}\left(2 a^{2}-14 a-8 c^{2}+64 c-108\right)\right] \\
& { }_{2} F_{1}\left[\begin{array}{ccc}
a, & 8-a ; & \frac{1}{2} \\
c & ; & =
\end{array}=\right. \\
& =\frac{\sqrt{\pi} \Gamma(c)}{2^{c-8}\left\{\prod_{\xi=1}^{7}(\xi-a)\right\}}\left[\frac { 1 } { \Gamma ( \frac { c - a } { 2 } ) \Gamma ( \frac { c + a - 7 } { 2 } ) } \left(-a^{3}-4 a^{2} c+30 a^{2}+4 a c^{2}-4 a c-107 a+8 c^{3}\right.\right. \\
& \left.-124 c^{2}+576 c-762\right)+ \\
& +\frac{1}{\Gamma\left(\frac{c-a+1}{2}\right) \Gamma\left(\frac{c+a-8}{2}\right)}\left(-a^{3}+4 a^{2} c-6 a^{2}+4 a c^{2}-68 a c+181 a\right. \\
& \left.\left.-8 c^{3}+92 c^{2}-288 c+210\right)\right] \\
& { }_{2} F_{1}\left[\begin{array}{ccc}
a, & 9-a ; & \frac{1}{2} \\
c & ; & =
\end{array}=\right. \\
& =\frac{\sqrt{\pi} \Gamma(c)}{2^{c-9}\left\{\prod_{\varpi=1}^{8}(\varpi-a)\right\}}\left[\frac { 1 } { \Gamma ( \frac { c - a + 1 } { 2 } ) \Gamma ( \frac { c + a - 8 } { 2 } ) } \left(a^{4}-18 a^{3}-8 a^{2} c^{2}+80 a^{2} c-85 a^{2}+72 a c^{2}\right.\right. \\
& -720 a c+1494 a+8 c^{4}- \\
& \left.-160 c^{3}+1056 c^{2}-2560 c+1680\right)+\frac{1}{\Gamma\left(\frac{c-a}{2}\right) \Gamma\left(\frac{c+a-9}{2}\right)}\left(8 a^{2} c\right. \\
& -40 a^{2}-72 a c+360 a-16 c^{3}+240 c^{2}-1072 c \\
& +1360)] \\
& { }_{2} F_{1}\left[\begin{array}{ccc}
a, & 10-a ; & \frac{1}{2} \\
c & ; & =
\end{array}\right]= \\
& =\frac{\sqrt{\pi} \Gamma(c)}{2^{c-10}\left\{\prod_{v=1}^{9}(v-a)\right\}}\left[\frac { 1 } { \Gamma ( \frac { c - a + 1 } { 2 } ) \Gamma ( \frac { c + a - 1 0 } { 2 } ) } \left(-a^{4}-4 a^{3} c+42 a^{3}+12 a^{2} c^{2}-72 a^{2} c-107 a^{2}\right.\right. \\
& +8 a c^{3}-252 a c^{2}+ \\
& \left.+1772 a c-3054 a-16 c^{4}+312 c^{3}-2000 c^{2}+4704 c-3024\right)+\frac{1}{\Gamma\left(\frac{c-a}{2}\right) \Gamma\left(\frac{c+a-9}{2}\right)}\left(a^{4}-4 a^{3} c\right. \\
& +2 a^{3}-12 a^{2} c^{2}+192 a^{2} c- \\
& -553 a^{2}+8 a c^{3}-12 a c^{2}-868 a c+3406 a+16 c^{4}-392 c^{3} \\
& \left.\left.+3320 c^{2}-11224 c+12264\right)\right] \\
& { }_{2} F_{1}\left[\begin{array}{ccc}
a, & 11-a ; & \frac{1}{2} \\
c & ; & =
\end{array}\right.
\end{aligned}
$$




$$
\begin{aligned}
& =\frac{\sqrt{\pi} \Gamma(c)}{2^{c-11}\left\{\prod_{\varphi=1}^{10}(\varphi-a)\right\}}\left[\frac { 1 } { \Gamma ( \frac { c - a + 1 } { 2 } ) \Gamma ( \frac { c + a - 1 0 } { 2 } ) } \left(5 a^{4} c-30 a^{4}-110 a^{3} c+660 a^{3}-20 a^{2} c^{3}\right.\right. \\
& +360 a^{2} c^{2}-1305 a^{2} c- \\
& -810 a^{2}+220 a c^{3}-3960 a c^{2}+21010 a c-31020 a+16 c^{5}-480 c^{4}+5240 c^{3}-25200 c^{2} \\
& +50544 c-30240)+ \\
& +\frac{1}{\Gamma\left(\frac{c-a}{2}\right) \Gamma\left(\frac{c+a-11}{2}\right)}\left(-2 a^{4}+44 a^{3}+24 a^{2} c^{2}-288 a^{2} c+530 a^{2}-264 a c^{2}+3168 a c-8492 a\right. \\
& -32 c^{4}+768 c^{3}-6352 c^{2}+ \\
& +20928 c-22320)] \\
& { }_{2} F_{1}\left[\begin{array}{ccc}
a, & 12-a ; & \frac{1}{2} \\
c & ; & =
\end{array}\right. \\
& =\frac{\sqrt{\pi} \Gamma(c)}{2^{c-12}\left\{\prod_{\chi=1}^{11}(\chi-a)\right\}}\left[\frac { 1 } { \Gamma ( \frac { c - a + 1 } { 2 } ) \Gamma ( \frac { c + a - 1 2 } { 2 } ) } \left(a^{5}-6 a^{4} c+9 a^{4}-12 a^{3} c^{2}+300 a^{3} c-1103 a^{3}\right.\right. \\
& +32 a^{2} c^{3}- \\
& -408 a^{2} c^{2}+46 a^{2} c+6351 a^{2}+16 a c^{4}-800 a c^{3}+10364 a c^{2}-46852 a c+62182 a-32 c^{5} \\
& +944 c^{4}-10112 c^{3}+47656 c^{2}- \\
& -93776 c+55440)+\frac{1}{\Gamma\left(\frac{c-a}{2}\right) \Gamma\left(\frac{c+a-11}{2}\right)}\left(a^{5}+6 a^{4} c-69 a^{4}-12 a^{3} c^{2}+12 a^{3} c+769 a^{3}\right. \\
& -32 a^{2} c^{3}+840 a^{2} c^{2}- \\
& -5662 a^{2} c+8301 a^{2}+16 a c^{4}-32 a c^{3}-4612 a c^{2}+42380 a c-96002 a+32 c^{5}-1136 c^{4} \\
& +15104 c^{3}- \\
& \left.\left.-92536 c^{2}+255392 c-245640\right)\right] \\
& { }_{2} F_{1}\left[\begin{array}{ccc}
a, & 13-a ; & \frac{1}{2} \\
c & ; & =
\end{array}\right. \\
& =\frac{\sqrt{\pi} \Gamma(c)}{2^{c-13}\left\{\prod_{\beta=1}^{12}(\beta-a)\right\}}\left[\frac { 1 } { \Gamma ( \frac { c - a + 1 } { 2 } ) \Gamma ( \frac { c + a - 1 2 } { 2 } ) } \left(-a^{6}+39 a^{5}+18 a^{4} c^{2}-252 a^{4} c+275 a^{4}\right.\right. \\
& -468 a^{3} c^{2}+6552 a^{3} c \\
& -18135 a^{3}-48 a^{2} c^{4}+1344 a^{2} c^{3}-9834 a^{2} c^{2}+5964 a^{2} c+74246 a^{2}+624 a c^{4}-17472 a c^{3} \\
& +167388 a c^{2}-631176 a c \\
& \left.+752856 a+32 c^{6}-1344 c^{5}+21824 c^{4}-172032 c^{3}+674384 c^{2}-1187424 c+665280\right)+
\end{aligned}
$$




$$
\begin{aligned}
& +\frac{1}{\Gamma\left(\frac{c-a}{2}\right) \Gamma\left(\frac{c+a-13}{2}\right)}\left(-12 a^{4} c+84 a^{4}+312 a^{3} c-2184 a^{3}+64 a^{2} c^{3}-1344 a^{2} c^{2}\right. \\
& +6620 a^{2} c-2436 a^{2}-832 a c^{3}+17472 a c^{2}-112424 a c+216216 a-64 c^{5}+2240 c^{4} \\
& -29312 c^{3}+176512 c^{2}- \\
& -478752 c+453600)] \\
& { }_{2} F_{1}\left[\begin{array}{ccc}
a, & 14-a ; & \frac{1}{2} \\
c & ; & =
\end{array}\right. \\
& =\frac{\sqrt{\pi} \Gamma(c)}{2^{c-14}\left\{\prod_{\sigma=1}^{13}(\sigma-a)\right\}}\left[\frac { 1 } { \Gamma ( \frac { c - a + 1 } { 2 } ) \Gamma ( \frac { c + a - 1 4 } { 2 } ) } \left(a^{6}+6 a^{5} c-87 a^{5}-24 a^{4} c^{2}+150 a^{4} c+925 a^{4}\right.\right. \\
& -32 a^{3} c^{3}+1392 a^{3} c^{2} \\
& -12706 a^{3} c+24615 a^{3}+80 a^{2} c^{4}-1728 a^{2} c^{3}+5368 a^{2} c^{2}+58986 a^{2} c-242486 a^{2}+32 a c^{5} \\
& -2320 a c^{4}+47328 a c^{3} \\
& -391568 a c^{2}+1344076 a c-1496568 a-64 c^{6}+2656 c^{5}-42560 c^{4}+330752 c^{3} \\
& \left.-1278144 c^{2}+2222160 c-1235520\right)+ \\
& +\frac{1}{\Gamma\left(\frac{c-a}{2}\right) \Gamma\left(\frac{c+a-13}{2}\right)}\left(-a^{6}+6 a^{5} c-\right. \\
& -3 a^{5}+24 a^{4} c^{2}-570 a^{4} c+2225 a^{4}-32 a^{3} c^{3}+48 a^{3} c^{2}+7454 a^{3} c-39225 a^{3}-80 a^{2} c^{4} \\
& +3072 a^{2} c^{3}-35608 a^{2} c^{2}+133626 a^{2} c- \\
& -68104 a^{2}+32 a c^{5}-80 a c^{4}-19872 a c^{3}+313808 a c^{2}-1676564 a c+2856228 a+64 c^{6} \\
& -3104 c^{5}+59360 c^{4}-566848 c^{3}+ \\
& \left.\left.+2810304 c^{2}-6724560 c+5897520\right)\right] \\
& { }_{2} F_{1}\left[\begin{array}{ccc}
a, & 15-a ; & \frac{1}{2} \\
c & ; & =
\end{array}\right]= \\
& =\frac{\sqrt{\pi} \Gamma(c)}{2^{c-15}\left\{\prod_{\varepsilon=1}^{14}(\varepsilon-a)\right\}}\left[\frac { 1 } { \Gamma ( \frac { c - a + 1 } { 2 } ) \Gamma ( \frac { c + a - 1 4 } { 2 } ) } \left(-7 a^{6} c+56 a^{6}+315 a^{5} c-2520 a^{5}+56 a^{4} c^{3}\right.\right. \\
& -1344 a^{4} c^{2}+5103 a^{4} c+ \\
& +16520 a^{4}-1680 a^{3} c^{3}+40320 a^{3} c^{2}-271215 a^{3} c+449400 a^{3}-112 a^{2} c^{5}+4480 a^{2} c^{4} \\
& -54040 a^{2} c^{3}+150080 a^{2} c^{2}+845824 a^{2} c- \\
& -3383296 a^{2}+1680 a c^{5}-67200 a c^{4}+999600 a c^{3}-6787200 a c^{2}+20482140 a c \\
& -21070560 a+64 c^{7}-3584 c^{6}+80864 c^{5}-
\end{aligned}
$$




$$
\begin{gathered}
\left.-940800 c^{4}+5987520 c^{3}-20296192 c^{2}+32464368 c-17297280\right) \\
+\frac{1}{\Gamma\left(\frac{c-a}{2}\right) \Gamma\left(\frac{c+a-15}{2}\right)}\left(2 a^{6}-90 a^{5}-48 a^{4} c^{2}+768 a^{4} c-\right. \\
-1474 a^{4}+1440 a^{3} c^{2}-23040 a^{3} c+77970 a^{3}+160 a^{2} c^{4}-5120 a^{2} c^{3}+46640 a^{2} c^{2} \\
-90880 a^{2} c-226192 a^{2}-2400 a c^{4}+
\end{gathered}
$$




$$
\begin{gathered}
=\frac{\sqrt{\pi} \Gamma(c)}{2^{c-17}\left\{\prod_{\vartheta=1}^{16}(\vartheta-a)\right\}}\left[\frac { 1 } { \Gamma ( \frac { c - a + 1 } { 2 } ) \Gamma ( \frac { c + a - 1 6 } { 2 } ) } \left(a^{8}-68 a^{7}-32 a^{6} c^{2}+576 a^{6} c-638 a^{6}\right.\right. \\
+1632 a^{5} c^{2}-29376 a^{5} c+101320 a^{5}+
\end{gathered}
$$

$+160 a^{4} c^{4}-5760 a^{4} c^{3}+44640 a^{4} c^{2}+129600 a^{4} c-1341071 a^{4}-5440 a^{3} c^{4}+195840 a^{3} c^{3}$ $-2303840 a^{3} c^{2}+$

$+9743040 a^{3} c-9832052 a^{3}-256 a^{2} c^{6}+13824 a^{2} c^{5}-246560 a^{2} c^{4}+1411200 a^{2} c^{3}$ $+4297408 a^{2} c^{2}-64103040 a^{2} c+$

$+143207628 a^{2}+4352 a c^{6}-235008 a c^{5}+4977600 a c^{4}-52289280 a c^{3}+282566656 a c^{2}$ $-727036416 a c+670152240 a+$

$$
\begin{gathered}
+128 c^{8}-9216 c^{7}+275456 c^{6}-4423680 c^{5}+41249792 c^{4}-224907264 c^{3} \\
+683065344 c^{2}-1014128640 c+518918400+
\end{gathered}
$$

$+\frac{1}{\Gamma\left(\frac{c-a}{2}\right) \Gamma\left(\frac{c+a-17}{2}\right)}\left(16 a^{6} c-144 a^{6}-816 a^{5} c+7344 a^{5}-160 a^{4} c^{3}+4320 a^{4} c^{2}-22480 a^{4} c\right.$ $-30960 a^{4}+5440 a^{3} c^{3}$

$-146880 a^{3} c^{2}+1157360 a^{3} c-2484720 a^{3}+384 a^{2} c^{5}-17280 a^{2} c^{4}+247840 a^{2} c^{3}$ $-1092960 a^{2} c^{2}-1901760 a^{2} c+$

$+15669504 a^{2}-6528 a c^{5}+293760 a c^{4}-4999360 a c^{3}+39804480 a c^{2}-146267456 a c$ $+194890176 a-256 c^{7}+16128 c^{6}-$

$-414976 c^{5}+5610240 c^{4}-42628864 c^{3}+179788032 c^{2}$

$-383195904 c+310867200)]$

$$
{ }_{2} F_{1}\left[\begin{array}{ccc}
a, & 18-a ; & \frac{1}{2} \\
c & ; & =
\end{array}\right]=
$$

$$
\begin{gathered}
=\frac{\sqrt{\pi} \Gamma(c)}{2^{c-18}\left\{\prod_{\eta=1}^{17}(\eta-a)\right\}}\left[\frac { 1 } { \Gamma ( \frac { c - a + 1 } { 2 } ) \Gamma ( \frac { c + a - 1 8 } { 2 } ) } \left(-a^{8}-8 a^{7} c+148 a^{7}+40 a^{6} c^{2}-256 a^{6} c\right.\right. \\
-3362 a^{6}+80 a^{5} c^{3}-4440 a^{5} c^{2}+
\end{gathered}
$$

$$
\begin{gathered}
+49664 a^{5} c-103400 a^{5}-240 a^{4} c^{4}+5520 a^{4} c^{3}+18760 a^{4} c^{2}-849520 a^{4} c+3240271 a^{4} \\
-192 a^{3} c^{5}+17760 a^{3} c^{4}-440560 a^{3} c^{3}+ \\
+4091160 a^{3} c^{2}-12923320 a^{3} c+3622852 a^{3}+448 a^{2} c^{6}-20352 a^{2} c^{5}+253360 a^{2} c^{4} \\
+576240 a^{2} c^{3}-31091248 a^{2} c^{2}+ \\
+192701168 a^{2} c-344444908 a^{2}+128 a c^{7}-16576 a c^{6}+660032 a c^{5}-12228640 a c^{4} \\
+118499872 a c^{3}-604789504 a c^{2}+ \\
+1488844864 a c-1324543920 a-256 c^{8}+18304 c^{7}-542976 c^{6}+8650240 c^{5} \\
-79993344 c^{4}+432549376 c^{3}-1303568384 c^{2}+
\end{gathered}
$$




$$
\begin{aligned}
& +1923025920 c-980179200)+\frac{1}{\Gamma\left(\frac{c-a}{2}\right) \Gamma\left(\frac{c+a-17}{2}\right)}\left(a^{8}-8 a^{7} c+4 a^{7}-40 a^{6} c^{2}+1264 a^{6} c\right. \\
& -6214 a^{6}+80 a^{5} c^{3}-120 a^{5} c^{2}- \\
& -32416 a^{5} c+213904 a^{5}+240 a^{4} c^{4}-12720 a^{4} c^{3}+186440 a^{4} c^{2}-743120 a^{4} c-456391 a^{4} \\
& -192 a^{3} c^{5}+480 a^{3} c^{4}+216080 a^{3} c^{3}- \\
& -4278120 a^{3} c^{2}+27569480 a^{3} c-52277444 a^{3}-448 a^{2} c^{6}+30720 a^{2} c^{5}-745840 a^{2} c^{4} \\
& +7817520 a^{2} c^{3}-30345632 a^{2} c^{2}- \\
& -19224224 a^{2} c+253516684 a^{2}+128 a c^{7}-448 a c^{6}-259264 a c^{5}+8556320 a c^{4} \\
& -118218848 a c^{3}+813195488 a c^{2}- \\
& -2692403360 a c+3335839536 a+256 c^{8}-20608 c^{7}+696192 c^{6}-12817024 c^{5} \\
& +139638144 c^{4}-913535872 c^{3}+3463541888 c^{2}- \\
& -6848013696 c+5284782720)] \\
& { }_{2} F_{1}\left[\begin{array}{ccc}
a, & 19-a ; & \frac{1}{2} \\
c & ; & =
\end{array}\right. \\
& =\frac{\sqrt{\pi} \Gamma(c)}{2^{c-19}\left\{\prod_{\lambda=1}^{18}(\lambda-a)\right\}}\left[\frac { 1 } { \Gamma ( \frac { c - a + 1 } { 2 } ) \Gamma ( \frac { c + a - 1 8 } { 2 } ) } \left(9 a^{8} c-90 a^{8}-684 a^{7} c+6840 a^{7}-120 a^{6} c^{3}\right.\right. \\
& +3600 a^{6} c^{2}-14046 a^{6} c-99540 a^{6}+ \\
& +6840 a^{5} c^{3}-205200 a^{5} c^{2}+1664856 a^{5} c-2968560 a^{5}+432 a^{4} c^{5}-21600 a^{4} c^{4} \\
& +277080 a^{4} c^{3}+327600 a^{4} c^{2}-20793831 a^{4} c+ \\
& +70898310 a^{4}-16416 a^{3} c^{5}+820800 a^{3} c^{4}-14644440 a^{3} c^{3}+111013200 a^{3} c^{2} \\
& -315518940 a^{3} c+131909400 a^{3}-576 a^{2} c^{7}+ \\
& +40320 a^{2} c^{6}-992880 a^{2} c^{5}+9324000 a^{2} c^{4}+4429536 a^{2} c^{3}-636886080 a^{2} c^{2} \\
& +3695816316 a^{2} c-6211091160 a^{2}+10944 a c^{7}- \\
& -766080 a c^{6}+21827808 a c^{5}-325310400 a c^{4}+2707726176 a c^{3}-12394025280 a c^{2} \\
& +28254838896 a c-23908836960 a+256 c^{9}- \\
& -23040 c^{8}+880512 c^{7}-18627840 c^{6}+238347264 c^{5}-1891123200 c^{4}+9158978048 c^{3} \\
& -25507261440 c^{2}+35661692160 c- \\
& -17643225600)+\frac{1}{\Gamma\left(\frac{c-a}{2}\right) \Gamma\left(\frac{c+a-19}{2}\right)}\left(-2 a^{8}+152 a^{7}+80 a^{6} c^{2}-1600 a^{6} c+3148 a^{6}\right. \\
& -4560 a^{5} c^{2}+91200 a^{5} c-371488 a^{5}- \\
& -480 a^{4} c^{4}+19200 a^{4} c^{3}-185680 a^{4} c^{2}-126400 a^{4} c+4559182 a^{4}+18240 a^{3} c^{4} \\
& -729600 a^{3} c^{3}+9799440 a^{3} c^{2}-50068800 a^{3} c+
\end{aligned}
$$




$$
\begin{aligned}
& +73373288 a^{3}+896 a^{2} c^{6}-53760 a^{2} c^{5}+1107680 a^{2} c^{4}-8467200 a^{2} c^{3}-743936 a^{2} c^{2} \\
& +274718720 a^{2} c-822056088 a^{2}- \\
& -17024 a c^{6}+1021440 a c^{5}-24338240 a c^{4}+292569600 a c^{3}-1853708096 a c^{2} \\
& +5798641920 a c-6885423072 a- \\
& -512 c^{8}+40960 c^{7}-1374464 c^{6}+25123840 c^{5}-271685888 c^{4}+1764075520 c^{3} \\
& -6639757056 c^{2}+13042437120 c- \\
& -10013310720)] \\
& { }_{2} F_{1}\left[\begin{array}{ccc}
a, & 20-a ; & \frac{1}{2} \\
c & ; & =
\end{array}\right. \\
& =\frac{\sqrt{\pi} \Gamma(c)}{2^{c-20}\left\{\prod_{Y=1}^{19}(Y-a)\right\}}\left[\frac{1}{\Gamma\left(\frac{c-a+1}{2}\right) \Gamma\left(\frac{c+a-20}{2}\right)}(33522128640+47215599696 a\right. \\
& +14182895460 a^{2}+345040520 a^{3}- \\
& -140133105 a^{4}+962073 a^{5}+330750 a^{6}-9330 a^{7}+15 a^{8}+a^{9}-67958134272 c \\
& -57343402272 a c-9605975576 a^{2} c+ \\
& +295428296 a^{3} c+58846422 a^{4} c-2100880 a^{5} c-32820 a^{6} c+1640 a^{7} c-10 a^{8} c \\
& +48842214912 c^{2}+ \\
& +25998562336 a c^{2}+2187966784 a^{2} c^{2}-168954152 a^{3} c^{2}-6101120 a^{4} c^{2}+380720 a^{5} c^{2} \\
& -2240 a^{6} c^{2}-40 a^{7} c^{2}-17641896960 c^{3}- \\
& -5917427456 a c^{3}-182014144 a^{2} c^{3}+27821280 a^{3} c^{3}-9440 a^{4} c^{3}-19680 a^{5} c^{3}+160 a^{6} c^{3} \\
& +3666323456 c^{4}+750095264 a c^{4}- \\
& -1895280 a^{2} c^{4}-1926160 a^{3} c^{4}+23280 a^{4} c^{4}+240 a^{5} c^{4}-465172736 c^{5}-54369728 a c^{5} \\
& +1155616 a^{2} c^{5}+55104 a^{3} c^{5}-672 a^{4} c^{5}+ \\
& +36595328 c^{6}+2174144 a c^{6}-61824 a^{2} c^{6}-448 a^{3} c^{6}-1740800 c^{7}-41984 a c^{7} \\
& \left.+1024 a^{2} c^{7}+45824 c^{8}+256 a c^{8}-512 c^{9}\right)+ \\
& +\frac{1}{\Gamma\left(\frac{c-a}{2}\right) \Gamma\left(\frac{c+a-19}{2}\right)}\left(-190253266560-131460917904 a-15315714660 a^{2}\right. \\
& +1718684120 a^{3}+100625805 a^{4}-10839927 a^{5}+ \\
& +135450 a^{6}+7470 a^{7}-195 a^{8}+a^{9}+258458522112 c+117489033888 a c \\
& +5199265016 a^{2} c-1259577944 a^{3} c+961578 a^{4} c+ \\
& +3256720 a^{5} c-84780 a^{6} c+40 a^{7} c+10 a^{8} c-139931759232 c^{2}-40815588704 a c^{2} \\
& +198370336 a^{2} c^{2}+283436248 a^{3} c^{2}-
\end{aligned}
$$




$$
\begin{aligned}
& -7330880 a^{4} c^{2}-224080 a^{5} c^{2}+7840 a^{6} c^{2}-40 a^{7} c^{2}+40472263680 c^{3}+7213462784 a c^{3} \\
& -274206656 a^{2} c^{3}-26053920 a^{3} c^{3}+ \\
& +1017440 a^{4} c^{3}-480 a^{5} c^{3}-160 a^{6} c^{3}-6993636736 c^{4}-700147936 a c^{4}+42392880 a^{2} c^{4} \\
& +896240 a^{3} c^{4}-47280 a^{4} c^{4}+240 a^{5} c^{4}+ \\
& +757008896 c^{5}+36475712 a c^{5}-2849056 a^{2} c^{5}+1344 a^{3} c^{5}+672 a^{4} c^{5}-51764608 c^{6} \\
& -836416 a c^{6}+88704 a^{2} c^{6}-448 a^{3} c^{6}+ \\
& +2170880 c^{7}-1024 a c^{7}-1024 a^{2} c^{7}-50944 c^{8}+256 a c^{8} \\
& \left.\left.+512 c^{9}\right)\right] \\
& { }_{2} F_{1}\left[\begin{array}{ccc}
a, & 21-a ; & \frac{1}{2} \\
c & ; & =
\end{array}\right]= \\
& =\frac{\sqrt{\pi} \Gamma(c)}{2^{c-21}\left\{\prod_{\Psi=1}^{20}(\Psi-a)\right\}}\left[\frac{1}{\Gamma\left(\frac{c-a+1}{2}\right) \Gamma\left(\frac{c+a-20}{2}\right)}(670442572800+946321185600 a\right. \\
& +284169369024 a^{2}+4885689900 a^{3}- \\
& -3333875180 a^{4}+41694345 a^{5}+10037727 a^{6}-381150 a^{7}+1230 a^{8}+105 a^{9}-a^{10} \\
& -1394694005760 c-1198379286720 a c- \\
& -203053089360 a^{2} c+8433107760 a^{3} c+1530533620 a^{4} c-70408800 a^{5} c-1146200 a^{6} c \\
& +92400 a^{7} c-1100 a^{8} c+ \\
& +1048586614272 c^{2}+578478838560 a c^{2}+49539606520 a^{2} c^{2}-4805882760 a^{3} c^{2} \\
& -177714670 a^{4} c^{2}+15397200 a^{5} c^{2}- \\
& -141500 a^{6} c^{2}-4200 a^{7} c^{2}+50 a^{8} c^{2}-404078540800 c^{3}-143591669760 a c^{3} \\
& -4354528640 a^{2} c^{3}+902932800 a^{3} c^{3}- \\
& -2094400 a^{4} c^{3}-1108800 a^{5} c^{3}+17600 a^{6} c^{3}+91700259840 c^{4}+20464187520 a c^{4} \\
& -122473120 a^{2} c^{4}-77439600 a^{3} c^{4}+ \\
& +1402800 a^{4} c^{4}+25200 a^{5} c^{4}-400 a^{6} c^{4}-13092907520 c^{5}-1739633280 a c^{5} \\
& +50240960 a^{2} c^{5}+3104640 a^{3} c^{5}-73920 a^{4} c^{5}+ \\
& +1209103616 c^{6}+87071040 a c^{6}-3652320 a^{2} c^{6}-47040 a^{3} c^{6}+1120 a^{4} c^{6}-72089600 c^{7} \\
& -2365440 a c^{7}+112640 a^{2} c^{7}+ \\
& \left.+2677760 c^{8}+26880 a c^{8}-1280 a^{2} c^{8}-56320 c^{9}+512 c^{10}\right) \\
& +\frac{1}{\Gamma\left(\frac{c-a}{2}\right) \Gamma\left(\frac{c+a-21}{2}\right)}(362387520000+268742591040 a+ \\
& +41471452880 a^{2}-1867829040 a^{3}-305673060 a^{4}+14303520 a^{5}+225720 a^{6} \\
& -18480 a^{7}+220 a^{8}-494250063360 c-
\end{aligned}
$$




$$
\begin{aligned}
& -247867413696 a c-19713479280 a^{2} c+1984361232 a^{3} c+69962284 a^{4} c-6179040 a^{5} c \\
& +56920 a^{6} c+1680 a^{7} c-20 a^{8} c+ \\
& +268936121344 c^{2}+89644203264 a c^{2}+2511762176 a^{2} c^{2}-547968960 a^{3} c^{2} \\
& +1404480 a^{4} c^{2}+665280 a^{5} c^{2}-10560 a^{6} c^{2}- \\
& -78226625536 c^{3}-16719935232 a c^{3}+112602112 a^{2} c^{3}+62139840 a^{3} c^{3}-1126720 a^{4} c^{3} \\
& -20160 a^{5} c^{3}+320 a^{6} c^{3}+ \\
& +13598953984 c^{4}+1756191360 a c^{4}-51029440 a^{2} c^{4}-3104640 a^{3} c^{4}+73920 a^{4} c^{4} \\
& -1480941056 c^{5}-104786304 a c^{5}+ \\
& +4397120 a^{2} c^{5}+56448 a^{3} c^{5}-1344 a^{4} c^{5}+101871616 c^{6}+3311616 a c^{6}-157696 a^{2} c^{6} \\
& -4296704 c^{7}-43008 a c^{7}+2048 a^{2} c^{7}+ \\
& \left.\left.+101376 c^{8}-1024 c^{9}\right)\right] \\
& { }_{2} F_{1}\left[\begin{array}{ccc}
a, & 22-a ; & \frac{1}{2} \\
c & ; & =
\end{array}\right. \\
& =\frac{\sqrt{\pi} \Gamma(c)}{2^{c-22}\left\{\prod_{E=1}^{21}(\Xi-a)\right\}}\left[\frac{1}{\Gamma\left(\frac{c-a+1}{2}\right) \Gamma\left(\frac{c+a-22}{2}\right)}(-1279935820800-1868233671360 a\right. \\
& -628352859744 a^{2}- \\
& -34417212780 a^{3}+5753119700 a^{4}+134236095 a^{5}-20700687 a^{6}+312270 a^{7}+8730 a^{8} \\
& -225 a^{9}+a^{10}+2668809669120 c+ \\
& +2417863186656 a c+489345655848 a^{2} c-219480864 a^{3} c-3388493178 a^{4} c \\
& +53042458 a^{5} c+4970700 a^{6} c-142820 a^{7} c+ \\
& +390 a^{8} c+10 a^{9} c-2014029186048 c^{2}-1197461040576 a c^{2}-138256171792 a^{2} c^{2} \\
& +5911683120 a^{3} c^{2}+583619652 a^{4} c^{2}- \\
& -22165920 a^{5} c^{2}-176120 a^{6} c^{2}+10800 a^{7} c^{2}-60 a^{8} c^{2}+779711413248 c^{3} \\
& +306554335232 a c^{3}+17513420736 a^{2} c^{3}- \\
& -1499242976 a^{3} c^{3}-32128320 a^{4} c^{3}+2199680 a^{5} c^{3}-13440 a^{6} c^{3}-160 a^{7} c^{3} \\
& -177857647616 c^{4}-45404661120 a c^{4}- \\
& -823493664 a^{2} c^{4}+154420560 a^{3} c^{4}-467600 a^{4} c^{4}-75600 a^{5} c^{4}+560 a^{6} c^{4} \\
& +25531683072 c^{5}+4062167872 a c^{5}- \\
& -27880608 a^{2} c^{5}-7519456 a^{3} c^{5}+86688 a^{4} c^{5}+672 a^{5} c^{5}-2370643968 c^{6} \\
& -219093504 a c^{6}+4625152 a^{2} c^{6}+161280 a^{3} c^{6}- \\
& -1792 a^{4} c^{6}+142098432 c^{7}+6765568 a c^{7}-178176 a^{2} c^{7}-1024 a^{3} c^{7}-5305344 c^{8} \\
& -103680 a c^{8}+2304 a^{2} c^{8}+112128 c^{9}+
\end{aligned}
$$




$$
\begin{aligned}
& \left.+512 a c^{9}-1024 c^{10}\right)+\frac{1}{\Gamma\left(\frac{c-a}{2}\right) \Gamma\left(\frac{c+a-21}{2}\right)}(7610141548800+5664039006240 a \\
& +862754799384 a^{2}-50618670580 a^{3}- \\
& -7467040370 a^{4}+438809595 a^{5}+6355587 a^{6}-793890 a^{7}+14040 a^{8}-5 a^{9}-a^{10} \\
& -10762094073600 c-5490993903456 a c- \\
& -428082370072 a^{2} c+53697863232 a^{3} c+1803933278 a^{4} c-214732742 a^{5} c \\
& +2793980 a^{6} c+100060 a^{7} c-2370 a^{8} c+10 a^{9} c+ \\
& +6167102701056 c^{2}+2126343680320 a c^{2}+53972779984 a^{2} c^{2}-16286791024 a^{3} c^{2} \\
& +92193948 a^{4} c^{2}+28580160 a^{5} c^{2}- \\
& -673960 a^{6} c^{2}+240 a^{7} c^{2}+60 a^{8} c^{2}-1923629552128 c^{3}-434298545536 a c^{3} \\
& +5057543680 a^{2} c^{3}+2145900064 a^{3} c^{3}- \\
& -52633280 a^{4} c^{3}-1200640 a^{5} c^{3}+38080 a^{6} c^{3}-160 a^{7} c^{3}+366720941312 c^{4} \\
& +51431687104 a c^{4}-1928215296 a^{2} c^{4}- \\
& -133374640 a^{3} c^{4}+4718000 a^{4} c^{4}-1680 a^{5} c^{4}-560 a^{6} c^{4}-45108419328 c^{5} \\
& -3603513536 a c^{5}+200868192 a^{2} c^{5}+ \\
& +3361568 a^{3} c^{5}-160608 a^{4} c^{5}+672 a^{5} c^{5}+3654604800 c^{6}+142266880 a c^{6} \\
& -10065664 a^{2} c^{6}+3584 a^{3} c^{6}+1792 a^{4} c^{6}- \\
& -193800192 c^{7}-2561024 a c^{7}+245760 a^{2} c^{7}-1024 a^{3} c^{7} \\
& +6471168 c^{8}-2304 a c^{8}-2304 a^{2} c^{8}-123392 c^{9} \\
& \left.\left.+512 a c^{9}+1024 c^{10}\right)\right] \\
& { }_{2} F_{1}\left[\begin{array}{ccc}
a, & 23-a ; & \frac{1}{2} \\
c & ; & =
\end{array}\right. \\
& =\frac{\sqrt{\pi} \Gamma(c)}{2^{c-23}\left\{\prod_{\Omega=1}^{22}(\Omega-a)\right\}}\left[\frac{1}{\Gamma\left(\frac{c-a+1}{2}\right) \Gamma\left(\frac{c+a-22}{2}\right)}(-28158588057600-41169473009280 a\right. \\
& -13846175136288 a^{2}- \\
& -688985043120 a^{3}+149373094200 a^{4}+2886127860 a^{5}-648260844 a^{6}+12963720 a^{7} \\
& +382800 a^{8}-15180 a^{9}+132 a^{10}+ \\
& +60062080780800 c+55217638146528 a c+11293160726232 a^{2} c-82022959260 a^{3} c \\
& -93645815450 a^{4} c+1995539777 a^{5} c+ \\
& +178029929 a^{6} c-6909430 a^{7} c+31460 a^{8} c+1265 a^{9} c-11 a^{1} 0 c-47111453908992 c^{2} \\
& -28820579344128 a c^{2}- \\
& -3392485386048 a^{2} c^{2}+183098051136 a^{3} c^{2}+17810133264 a^{4} c^{2}-867081600 a^{5} c^{2} \\
& -6985440 a^{6} c^{2}+728640 a^{7} c^{2}-7920 a^{8} c^{2}+
\end{aligned}
$$




$$
\begin{aligned}
& +19258856466432 c^{3}+7922696165824 a c^{3}+461791289168 a^{2} c^{3}-49584004624 a^{3} c^{3} \\
& -1091526436 a^{4} c^{3}+105693280 a^{5} c^{3}- \\
& -988680 a^{6} c^{3}-20240 a^{7} c^{3}+220 a^{8} c^{3}-4723308327936 c^{4}-1289002826496 a c^{4} \\
& -22320173568 a^{2} c^{4}+5914856640 a^{3} c^{4}- \\
& -30824640 a^{4} c^{4}-5100480 a^{5} c^{4}+73920 a^{6} c^{4}+745452131072 c^{5}+130485126464 a c^{5} \\
& -1364040832 a^{2} c^{5}-359725520 a^{3} c^{5}+ \\
& +6190800 a^{4} c^{5}+85008 a^{5} c^{5}-1232 a^{6} c^{5}-78371758080 c^{6}-8302221312 a c^{6} \\
& +235834368 a^{2} c^{6}+10881024 a^{3} c^{6}- \\
& -236544 a^{4} c^{6}+5546010624 c^{7}+322674176 a c^{7}-12539648 a^{2} c^{7}-129536 a^{3} c^{7} \\
& +2816 a^{4} c^{7}-260941824 c^{8}-6994944 a c^{8}+ \\
& \left.+304128 a^{2} c^{8}+7822848 c^{9}+64768 a c^{9}-2816 a^{2} c^{9}-135168 c^{10}+1024 c^{11}\right) \\
& +\frac{1}{\Gamma\left(\frac{c-a}{2}\right) \Gamma\left(\frac{c+a-23}{2}\right)}(-14558535129600- \\
& -11503844032320 a-2137013714928 a^{2}+23993967080 a^{3}+17223845140 a^{4} \\
& -382057830 a^{5}-32189094 a^{6}+1259940 a^{7}- \\
& -5760 a^{8}-230 a^{9}+2 a^{10}+20652447375360 c+11426734414848 a c \\
& +1249606186752 a^{2} c-70894693632 a^{3} c- \\
& -6453736128 a^{4} c+319011840 a^{5} c+2486400 a^{6} c-264960 a^{7} c+2880 a^{8} c \\
& -11881425202176 c^{2}-4559948772992 a c^{2}- \\
& -249358186400 a^{2} c^{2}+27700361696 a^{3} c^{2}+584111304 a^{4} c^{2}-57805440 a^{5} c^{2} \\
& +541520 a^{6} c^{2}+11040 a^{7} c^{2}-120 a^{8} c^{2}+ \\
& +3722781351936 c^{3}+967717718016 a c^{3}+15372177408 a^{2} c^{3}-4341281280 a^{3} c^{3} \\
& +23278080 a^{4} c^{3}+3709440 a^{5} c^{3}- \\
& -53760 a^{6} c^{3}-713155826176 c^{4}-120677707136 a c^{4}+1319899392 a^{2} c^{4} \\
& +327847520 a^{3} c^{4}-5645920 a^{4} c^{4}-77280 a^{5} c^{4}+ \\
& +1120 a^{6} c^{4}+88159518720 c^{5}+9128189952 a c^{5}-260370432 a^{2} c^{5}-11870208 a^{3} c^{5} \\
& +258048 a^{4} c^{5}-7178121216 c^{6}- \\
& -411665408 a c^{6}+16002560 a^{2} c^{6}+164864 a^{3} c^{6}-3584 a^{4} c^{6}+382500864 c^{7} \\
& +10174464 a c^{7}-442368 a^{2} c^{7}-12831744 c^{8}- \\
& \left.\left.-105984 a c^{8}+4608 a^{2} c^{8}+245760 c^{9}-2048 c^{10}\right)\right] \\
& { }_{2} F_{1}\left[\begin{array}{ccc}
a, & 24-a ; & \frac{1}{2} \\
c & ; & =
\end{array}\right]=
\end{aligned}
$$




$$
\begin{aligned}
& =\frac{\sqrt{\pi} \Gamma(c)}{2^{c-24}\left\{\prod_{v=1}^{23}(v-a)\right\}}\left[\frac{1}{\Gamma\left(\frac{c-a+1}{2}\right) \Gamma\left(\frac{c+a-24}{2}\right)}(53970627110400+81275748452640 a\right. \\
& +29965288299912 a^{2}+ \\
& +2450226723044 a^{3}-225124440810 a^{4}-14621050415 a^{5}+1046631306 a^{6} \\
& +3307857 a^{7}-1146390 a^{8}+19675 a^{9}-18 a^{10}- \\
& -a^{11}-115335576760320 c-110963670941184 a c-26002256223296 a^{2} c \\
& -677875698640 a^{3} c+179871679040 a^{4} c+ \\
& +1164213764 a^{5} c-418718356 a^{6} c+7202120 a^{7} c+106120 a^{8} c-2940 a^{9} c+12 a^{10} c \\
& +90736096819200 c^{2}+ \\
& +59116525645888 a c^{2}+8571136553968 a^{2} c^{2}-132498699168 a^{3} c^{2}-43219364300 a^{4} c^{2} \\
& +885926748 a^{5} c^{2}+ \\
& +41948200 a^{6} c^{2}-1232440 a^{7} c^{2}+4020 a^{8} c^{2}+60 a^{9} c^{2}-37229387082752 c^{3} \\
& -16647614537344 a c^{3}-1382871809056 a^{2} c^{3}+ \\
& +70301031136 a^{3} c^{3}+4193488488 a^{4} c^{3}-166658240 a^{5} c^{3}-636720 a^{6} c^{3}+54880 a^{7} c^{3} \\
& -280 a^{8} c^{3}+9168624153856 c^{4}+ \\
& +2788555498944 a c^{4}+110886499136 a^{2} c^{4}-10435825456 a^{3} c^{4}-136259200 a^{4} c^{4} \\
& +10643360 a^{5} c^{4}-64960 a^{6} c^{4}- \\
& -560 a^{7} c^{4}-1453428736000 c^{5}-292828010496 a c^{5}-2923085312 a^{2} c^{5} \\
& +743957760 a^{3} c^{5}-3395840 a^{4} c^{5}-263424 a^{5} c^{5}+ \\
& +1792 a^{6} c^{5}+153495164928 c^{6}+19572034048 a c^{6}-185488128 a^{2} c^{6}-27007232 a^{3} c^{6} \\
& +295680 a^{4} c^{6}+1792 a^{5} c^{6}- \\
& -10911129600 c^{7}-818236416 a c^{7}+16882176 a^{2} c^{7}+451584 a^{3} c^{7}-4608 a^{4} c^{7} \\
& +515633664 c^{8}+20126976 a c^{8}- \\
& -493056 a^{2} c^{8}-2304 a^{3} c^{8}-15523840 c^{9}-250880 a c^{9}+5120 a^{2} c^{9}+269312 c^{10} \\
& \left.+1024 a c^{10}-2048 c^{11}\right)+ \\
& \begin{array}{l}
+\frac{1}{\Gamma\left(\frac{c-a}{2}\right) \Gamma\left(\frac{c+a-23}{2}\right)}\left(-334846347897600-265325367396960 a-48906562552488 a^{2}\right. \\
\quad+988440027044 a^{3}+
\end{array} \\
& +456572472690 a^{4}-13283988815 a^{5}-1027452594 a^{6}+51835857 a^{7}-355890 a^{8} \\
& -16325 a^{9}+282 a^{10}-a^{11}+ \\
& +490301899960320 c+275033962166784 a c+29894874504896 a^{2} c \\
& -2110031722960 a^{3} c-182994379040 a^{4} c+
\end{aligned}
$$




$$
\begin{gathered}
+11313648836 a^{5} c+75158356 a^{6} c-13480120 a^{7} c+217880 a^{8} c-60 a^{9} c-12 a^{10} c \\
-294885357427200 c^{2}- \\
-116251083614912 a c^{2}-6231457365232 a^{2} c^{2}+863633176032 a^{3} c^{2} \\
+17220272300 a^{4} c^{2}-2360841252 a^{5} c^{2}+ \\
+30594200 a^{6} c^{2}+783560 a^{7} c^{2}-16980 a^{8} c^{2}+60 a^{9} c^{2}+97997531697152 c^{3} \\
+26592490242944 a c^{3}+357545095456 a^{2} c^{3}-
\end{gathered}
$$$$
-150694485536 a^{3} c^{3}+1256431512 a^{4} c^{3}+188722240 a^{5} c^{3}-4067280 a^{6} c^{3}+1120 a^{7} c^{3}
$$$$
+280 a^{8} c^{3}-20258796646144 c^{4}-
$$$$
-3653166613056 a c^{4}+56989635136 a^{2} c^{4}+13326094544 a^{3} c^{4}-308739200 a^{4} c^{4}
$$$$
-5484640 a^{5} c^{4}+159040 a^{6} c^{4}-560 a^{7} c^{4}+
$$$$
+2762273996800 c^{5}+313271863296 a c^{5}-11408255488 a^{2} c^{5}-603912960 a^{3} c^{5}
$$$$
+19523840 a^{4} c^{5}-5376 a^{5} c^{5}-1792 a^{6} c^{5}-
$$

$-255403395072 c^{6}-16677258752 a c^{6}+860412672 a^{2} c^{6}+11699968 a^{3} c^{6}-510720 a^{4} c^{6}$ $+1792 a^{5} c^{6}+16110796800 c^{7}+$

$+517641216 a c^{7}-33470976 a^{2} c^{7}+9216 a^{3} c^{7}+4608 a^{4} c^{7}-682830336 c^{8}-7521024 a c^{8}$ $+658944 a^{2} c^{8}-2304 a^{3} c^{8}+$$$
+18595840 c^{9}-5120 a c^{9}-5120 a^{2} c^{9}-293888 c^{10}+1024 a c^{10}
$$$$
\left.\left.+2048 c^{11}\right)\right]
$$

$$
{ }_{2} F_{1}\left[\begin{array}{lcc}
a, & 25-a ; & \frac{1}{2} \\
c & ; & =
\end{array}\right]=
$$

$$
\begin{gathered}
=\frac{\sqrt{\pi} \Gamma(c)}{2^{c-25}\left\{\prod_{\rho=1}^{24}(\rho-a)\right\}}\left[\frac{1}{\Gamma\left(\frac{c-a+1}{2}\right) \Gamma\left(\frac{c+a-24}{2}\right)}(1295295050649600+1953217026028800 a\right. \\
+720182075218848 a^{2}+
\end{gathered}
$$

$$
\begin{aligned}
&+56242392874200 a^{3}-6430140382484 a^{4}-384225527550 a^{5}+34503650159 a^{6} \\
&+ 18877950 a^{7}-48737217 a^{8}+ \\
&+1122750 a^{9}- 2107 a^{10}-150 a^{11}+a^{12}-2824623532523520 c-2750688728985600 a c \\
&- 650557782840576 a^{2} c-
\end{aligned}
$$

$-14031184440000 a^{3} c+5306549788800 a^{4} c+16204297200 a^{5} c-15185557296 a^{6} c$ $+337740000 a^{7} c+5397600 a^{8} c-$

$$
\begin{aligned}
-234000 a^{9} c & +1872 a^{10} c+2298271622344704 c^{2}+1532660626368000 a c^{2} \\
+ & 226240061985280 a^{2} c^{2}-
\end{aligned}
$$


$-5059473463200 a^{3} c^{2}-1376735080736 a^{4} c^{2}+36195503400 a^{5} c^{2}+1749343288 a^{6} c^{2}$ $-69774000 a^{7} c^{2}+360240 a^{8} c^{2}+$

$$
\begin{gathered}
+9000 a^{9} c^{2}-72 a^{10} c^{2}-988040333070336 c^{3}-458238106560000 a c^{3} \\
-39008403657600 a^{2} c^{3}+2443984233600 a^{3} c^{3}+
\end{gathered}
$$

$$
\begin{aligned}
& +148362815328 a^{4} c^{3}-7556640000 a^{5} c^{3}-26644800 a^{6} c^{3}+4368000 a^{7} c^{3}-43680 a^{8} c^{3} \\
& +258663765079040 c^{4}+ \\
& +82943231664000 a c^{4}+3361869113440 a^{2} c^{4}-398515370400 a^{3} c^{4}-5286817592 a^{4} c^{4} \\
& +599592000 a^{5} c^{4}-5544560 a^{6} c^{4}- \\
& -84000 a^{7} c^{4}+840 a^{8} c^{4}-44346659635200 c^{5}-9621229363200 a c^{5}-85147625472 a^{2} c^{5} \\
& +33231744000 a^{3} c^{5}- \\
& -227834880 a^{4} c^{5}-20966400 a^{5} c^{5}+279552 a^{6} c^{5}+5176704237568 c^{6} \\
& \quad+731346739200 a c^{6}-9600109568 a^{2} c^{6}-
\end{aligned}
$$

$-1516300800 a^{3} c^{6}+24726016 a^{4} c^{6}+268800 a^{5} c^{6}-3584 a^{6} c^{6}-418737586176 c^{7}$

$-36241920000 a c^{7}+1000396800 a^{2} c^{7}+$

$$
\begin{gathered}
+35942400 a^{3} c^{7}-718848 a^{4} c^{7}+23472279552 c^{8}+1127232000 a c^{8}-40769280 a^{2} c^{8} \\
-345600 a^{3} c^{8}+6912 a^{4} c^{8}-
\end{gathered}
$$$$
-894566400 c^{9}-19968000 a c^{9}+798720 a^{2} c^{9}+22110208 c^{10}+153600 a c^{10}-6144 a^{2} c^{10}
$$$$
\left.-319488 c^{11}+2048 c^{12}\right)+
$$$$
+\frac{1}{\Gamma\left(\frac{c-a}{2}\right) \Gamma\left(\frac{c+a-25}{2}\right)}\left(642905026283520+536430721132800 a+114941722154688 a^{2}\right.
$$$$
+1925655420000 a^{3}-
$$$$
-909114008400 a^{4}-1437781800 a^{5}+2535320424 a^{6}-57018000 a^{7}-892320 a^{8}
$$$$
+39000 a^{9}-312 a^{10}-
$$

$-943741088299008 c-566915213203200 a c-77644945591872 a^{2} c$

$$
+1953684829600 a^{3} c+462411503408 a^{4} c-
$$

$-12465164600 a^{5} c-579414472 a^{6} c+23314000 a^{7} c-120640 a^{8} c-3000 a^{9} c+24 a^{10} c$ $+569402217805824 c^{2}+$

$+245281402080000 a c^{2}+19642608356800 a^{2} c^{2}-1277504155200 a^{3} c^{2}$

$-73799166896 a^{4} c^{2}+3813264000 a^{5} c^{2}+$

$+12856480 a^{6} c^{2}-2184000 a^{7} c^{2}+21840 a^{8} c^{2}-189917707732992 c^{3}$

$-57743834144000 a c^{3}-2208865074240 a^{2} c^{3}+$

$+270734475200 a^{3} c^{3}+3442060496 a^{4} c^{3}-400624000 a^{5} c^{3}+3708320 a^{6} c^{3}+56000 a^{7} c^{3}$ $-560 a^{8} c^{3}+39416989081600 c^{4}+$ 


$$
\begin{aligned}
& +8229521664000 a c^{4}+65103933440 a^{2} c^{4}-27902784000 a^{3} c^{4}+194055680 a^{4} c^{4} \\
& +17472000 a^{5} c^{4}-232960 a^{6} c^{4}- \\
& -5396707745792 c^{5}-741874022400 a c^{5}+9980880896 a^{2} c^{5}+1519526400 a^{3} c^{5} \\
& -24790528 a^{4} c^{5}-268800 a^{5} c^{5}+ \\
& +3584 a^{6} c^{5}+501075714048 c^{6}+42561792000 a c^{6}-1178311680 a^{2} c^{6}-41932800 a^{3} c^{6} \\
& +838656 a^{4} c^{6}-31738847232 c^{7}- \\
& -1506048000 a c^{7}+54481920 a^{2} c^{7}+460800 a^{3} c^{7}-9216 a^{4} c^{7}+1350635520 c^{8} \\
& +29952000 a c^{8}-1198080 a^{2} c^{8}- \\
& -36925440 c^{9}-256000 a c^{9}+10240 a^{2} c^{9}+585728 c^{10} \\
& \left.\left.-4096 c^{11}\right)\right] \\
& { }_{2} F_{1}\left[\begin{array}{ccc}
a, 26-a ; & \frac{1}{2} \\
c & ; & =
\end{array}\right. \\
& =\frac{\sqrt{\pi} \Gamma(c)}{2^{c-26}\left\{\prod_{\tau=1}^{25}(\tau-a)\right\}}\left[\frac{1}{\Gamma\left(\frac{c-a+1}{2}\right) \Gamma\left(\frac{c+a-26}{2}\right)}(-2490952020480000-3856567328006400 a\right. \\
& -1535121422480160 a^{2}- \\
& -162685541616312 a^{3}+7957280277044 a^{4}+1100869544310 a^{5}-43694979215 a^{6} \\
& -1539415206 a^{7}+90397857 a^{8}- \\
& -769110 a^{9}-18725 a^{10}+318 a^{11}-a^{12}+5440375458201600 c+5512278582766080 a c \\
& +1453485267708864 a^{2} c+ \\
& +72388041958576 a^{3} c-9060718762800 a^{4} c-307607766540 a^{5} c+27726305208 a^{6} c \\
& -96424644 a^{7} c-19614240 a^{8} c+ \\
& +339500 a^{9} c-552 a^{10} c-12 a^{11} c-4437289667266560 c^{2}-3123375119467776 a c^{2} \\
& -539380387024256 a^{2} c^{2}- \\
& -4354450180400 a^{3} c^{2}+2864279730800 a^{4} c^{2}-8089061876 a^{5} c^{2}-4607346604 a^{6} c^{2} \\
& +84726040 a^{7} c^{2}+721000 a^{8} c^{2}- \\
& -22260 a^{9} c^{2}+84 a^{10} c^{2}+1913345384014848 c^{3}+952103060350336 a c^{3} \\
& +102944105640800 a^{2} c^{3}- \\
& -2607695699200 a^{3} c^{3}-390111231160 a^{4} c^{3}+9154149592 a^{5} c^{3}+261055760 a^{6} c^{3} \\
& -7910000 a^{7} c^{3}+27720 a^{8} c^{3}+ \\
& +280 a^{9} c^{3}-502609149259776 c^{4}-176315887270400 a c^{4}-10847680617600 a^{2} c^{4} \\
& +618019552640 a^{3} c^{4}+
\end{aligned}
$$




$$
\begin{aligned}
& +24394548640 a^{4} c^{4}-1011055360 a^{5} c^{4}-1400000 a^{6} c^{4}+237440 a^{7} c^{4}-1120 a^{8} c^{4} \\
& +86485139701760 c^{5}+ \\
& +21027657410560 a c^{5}+589073089024 a^{2} c^{5}-61326539008 a^{3} c^{5}-482101760 a^{4} c^{5} \\
& +45516800 a^{5} c^{5}-272384 a^{6} c^{5}- \\
& -1792 a^{7} c^{5}-10133908029440 c^{6}-1655765594112 a c^{6}-7453361664 a^{2} c^{6} \\
& +3226325760 a^{3} c^{6}-17606400 a^{4} c^{6}- \\
& -854784 a^{5} c^{6}+5376 a^{6} c^{6}+822856065024 c^{7}+86073947136 a c^{7}-952220160 a^{2} c^{7} \\
& -90969600 a^{3} c^{7}+944640 a^{4} c^{7}+ \\
& +4608 a^{5} c^{7}-46299721728 c^{8}-2875799040 a c^{8}+57504000 a^{2} c^{8}+1221120 a^{3} c^{8} \\
& -11520 a^{4} c^{8}+1771069440 c^{9}+ \\
& +57728000 a c^{9}-1320960 a^{2} c^{9}-5120 a^{3} c^{9}-43929600 c^{10}-596992 a c^{10}+11264 a^{2} c^{10} \\
& +636928 c^{11} \\
& \left.+2048 a c^{11}-4096 c^{12}\right)+\frac{1}{\Gamma\left(\frac{c-a}{2}\right) \Gamma\left(\frac{c+a-25}{2}\right)}(16072626136089600+13440284546567040 a \\
& +2865451725805248 a^{2}+ \\
& +29899479296856 a^{3}-26048131417484 a^{4}+79808986650 a^{5}+85137424259 a^{6} \\
& -2401982262 a^{7}-36821217 a^{8}+ \\
& +2318910 a^{9}-27607 a^{10}+6 a^{11}+a^{12}-24265950238448640 c-14741496935911296 a c \\
& -2017268942695968 a^{2} c+ \\
& +68446835425936 a^{3} c+14014027182600 a^{4} c-473895443004 a^{5} c-21308046816 a^{6} c \\
& +1112026236 a^{7} c- \\
& -8236440 a^{8} c-250180 a^{9} c+3984 a^{10} c-12 a^{11} c+15219075677764608 c^{2} \\
& +6700424924066688 a c^{2}+ \\
& +535657463741216 a^{2} c^{2}-42544045416400 a^{3} c^{2}-2402722747640 a^{4} c^{2} \\
& +155381269148 a^{5} c^{2}+343370524 a^{6} c^{2}- \\
& -129859240 a^{7} c^{2}+1932560 a^{8} c^{2}-420 a^{9} c^{2}-84 a^{10} c^{2}-5339375497061376 c^{3} \\
& -1680535240613504 a c^{3}- \\
& -62742622448800 a^{2} c^{3}+9664699301120 a^{3} c^{3}+112990802120 a^{4} c^{3}-19005822248 a^{5} c^{3} \\
& +240492560 a^{6} c^{3}+ \\
& +4669840 a^{7} c^{3}-93240 a^{8} c^{3}+280 a^{9} c^{3}+1181843678785536 c^{4}+259516705395200 a c^{4} \\
& +1441658770560 a^{2} c^{4}- \\
& -1119356174720 a^{3} c^{4}+11001976160 a^{4} c^{4}+1038876160 a^{5} c^{4}-20614720 a^{6} c^{4} \\
& +4480 a^{7} c^{4}+1120 a^{8} c^{4}-
\end{aligned}
$$


$-175498953625600 c^{5}-25896839035904 a c^{5}+463983127552 a^{2} c^{5}+71818487552 a^{3} c^{5}$ $-1570741760 a^{4} c^{5}-$

$-22414336 a^{5} c^{5}+598528 a^{6} c^{5}-1792 a^{7} c^{5}+18057365540864 c^{6}+1692014693376 a c^{6}$ $-59402198016 a^{2} c^{6}-$

$$
\begin{aligned}
& -2493308160 a^{3} c^{6}+74215680 a^{4} c^{6}-16128 a^{5} c^{6}-5376 a^{6} c^{6}-1304623362048 c^{7} \\
& -70987152384 a c^{7}+ \\
& +3406487040 a^{2} c^{7}+38423040 a^{3} c^{7}-1543680 a^{4} c^{7}+4608 a^{5} c^{7}+65999751168 c^{8} \\
& +1780938240 a c^{8}- \\
& -106026240 a^{2} c^{8}+23040 a^{3} c^{8}+11520 a^{4} c^{8}-2289039360 c^{9}-21345280 a c^{9} \\
& +1720320 a^{2} c^{9}-5120 a^{3} c^{9}+51836928 c^{10}- \\
& -11264 a c^{10}-11264 a^{2} c^{10}-690176 c^{11}+2048 a c^{11} \\
& \left.\left.+4096 c^{12}\right)\right] \\
& { }_{2} F_{1}\left[\begin{array}{ccc}
a, & 27-a ; & \frac{1}{2} \\
c & ; & =
\end{array}\right]= \\
& =\frac{\sqrt{\pi} \Gamma(c)}{2^{c-27}\left\{\prod_{\omega=1}^{26}(\omega-a)\right\}}\left[\frac{1}{\Gamma\left(\frac{c-a+1}{2}\right) \Gamma\left(\frac{c+a-26}{2}\right)}(-64764752532480000\right. \\
& -100380043171699200 a-
\end{aligned}
$$

$-39961833294277440 a^{2}-4123736953919856 a^{3}+256023569984728 a^{4}$

$$
+31801134144780 a^{5}-
$$

$-1558156952170 a^{6}-50373915228 a^{7}+3827623254 a^{8}-43587180 a^{9}-1136590 a^{10}$

$$
+29484 a^{11}-182 a^{12}+
$$

$+144050006577254400 c+147452461905565440 a c+39195512438330976 a^{2} c$ $+1832112082132488 a^{3} c-$

$-287018424824820 a^{4} c-8938296035370 a^{5} c+1036785419267 a^{6} c-6318393822 a^{7} c$ $-961728573 a^{8} c+$

$+22376250 a^{9} c-61503 a^{10} c-2106 a^{11} c+13 a^{12} c-121037881922979840 c^{2}$ $-86868374537887488 a c^{2}-$

$-15242604318923072 a^{2} c^{2}-55558110535200 a^{3} c^{2}+95763174889200 a^{4} c^{2}$ $-590522711688 a^{5} c^{2}-$

$-191785682088 a^{6} c^{2}+4514393520 a^{7} c^{2}+41787200 a^{8} c^{2}-2063880 a^{9} c^{2}+15288 a^{10} c^{2}$ $+54356751317087232 c^{3}+$ 


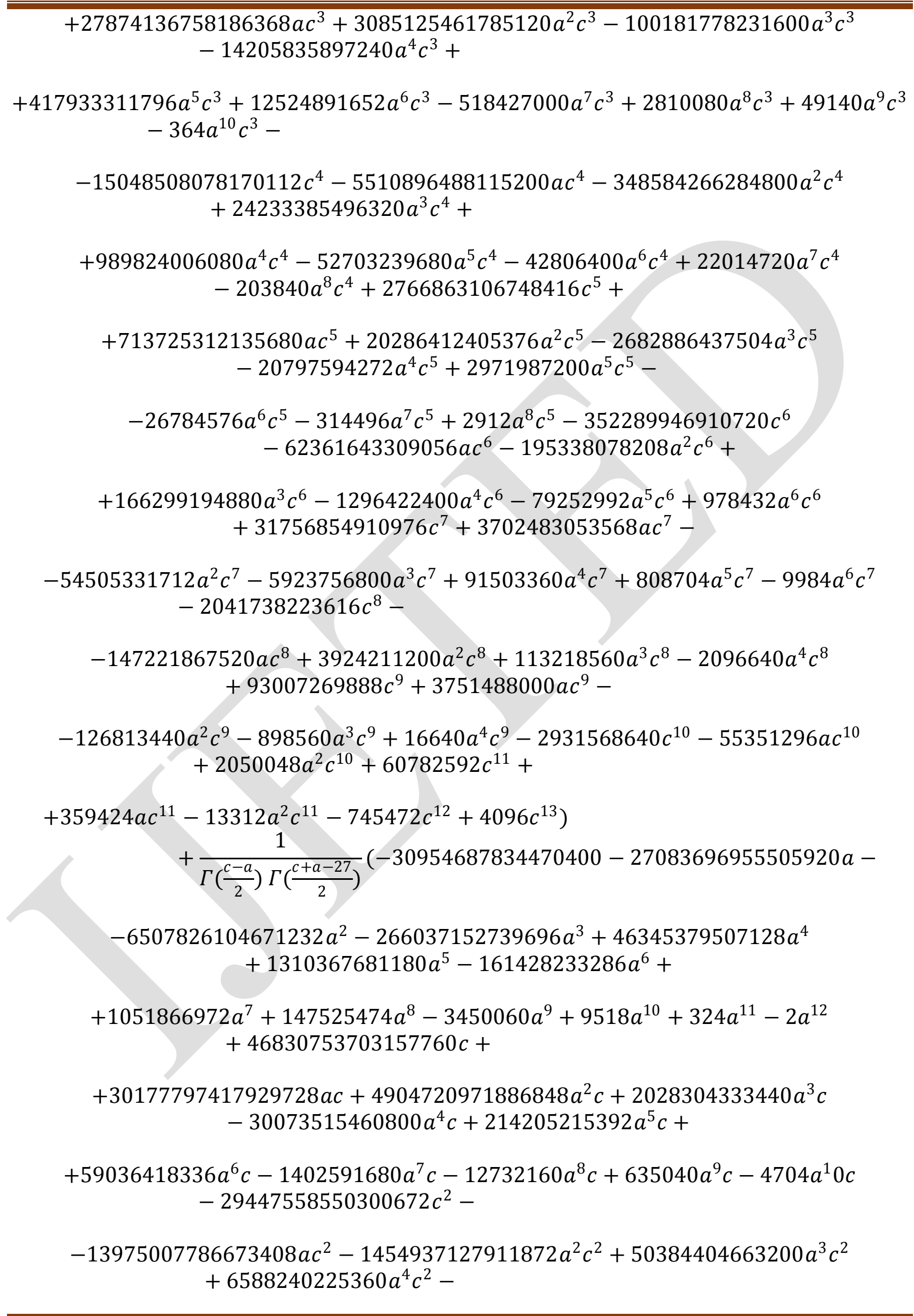


$-197467198488 a^{5} c^{2}-5739479928 a^{6} c^{2}+239757840 a^{7} c^{2}-1301440 a^{8} c^{2}-22680 a^{9} c^{2}$ $+168 a^{10} c^{2}+$

$+10362233929793536 c^{3}+3584472290058240 a c^{3}+215080588441600 a^{2} c^{3}$ $-15425517864960 a^{3} c^{3}-$

$-605115409920 a^{4} c^{3}+32676618240 a^{5} c^{3}+23331840 a^{6} c^{3}-13547520 a^{7} c^{3}$ $+125440 a^{8} c^{3}-2301160681971712 c^{4}-$

$-569028657484800 a c^{4}-15308983466240 a^{2} c^{4}+2095673368320 a^{3} c^{4}$

$+15505116480 a^{4} c^{4}-2290498560 a^{5} c^{4}+$

$+20657280 a^{6} c^{4}+241920 a^{7} c^{4}-2240 a^{8} c^{4}+342894825799680 c^{5}$ $+58835112763392 a c^{5}+146424004608 a^{2} c^{5}-$

$-154482370560 a^{3} c^{5}+1214760960 a^{4} c^{5}+73156608 a^{5} c^{5}-903168 a^{6} c^{5}$ $-35405951328256 c^{6}-4035795757056 a c^{6}+$

$+60337815552 a^{2} c^{6}+6391042560 a^{3} c^{6}-98757120 a^{4} c^{6}-870912 a^{5} c^{6}+10752 a^{6} c^{6}$ $+2567120683008 c^{7}+$

$+182218014720 a c^{7}-4867645440 a^{2} c^{7}-139345920 a^{3} c^{7}+2580480 a^{4} c^{7}$

$-130323050496 c^{8}-5203491840 a c^{8}+$

$+175925760 a^{2} c^{8}+1244160 a^{3} c^{8}-23040 a^{4} c^{8}+4535336960 c^{9}+85155840 a c^{9}$

$-3153920 a^{2} c^{9}-103043072 c^{10}-$

$\left.\left.-608256 a c^{10}+22528 a^{2} c^{10}+1376256 c^{11}-8192 c^{12}\right)\right]$

$$
{ }_{2} F_{1}\left[\begin{array}{ccc}
a, 28-a ; & \frac{1}{2} \\
c & ; & =
\end{array}\right.
$$

$=\frac{\sqrt{\pi} \Gamma(c)}{2^{c-28}\left\{\prod_{\varrho=1}^{27}(\varrho-a)\right\}}\left[\frac{1}{\Gamma\left(\frac{c-a+1}{2}\right) \Gamma\left(\frac{c+a-28}{2}\right)}(124903451312640000\right.$

$+198245272713580800 a+$

$+84237341463908640 a^{2}+10772713785416184 a^{3}-197837366197044 a^{4}$

$$
-74046181005314 a^{5}+1239432836775 a^{6}+
$$

$+162122858357 a^{7}-5261040477 a^{8}-33773187 a^{9}+3183285 a^{10}-36841 a^{11}+21 a^{12}$

$$
+a^{13}-278170295082854400 c-
$$

$-294899108770863360 a c-85743719979624576 a^{2} c-6115709513194896 a^{3} c$

$+428497731401864 a^{4} c+$

$+31572110867940 a^{5} c-1543488682402 a^{6} c-26614826532 a^{7} c+2007873630 a^{8} c$ $-19898340 a^{9} c-268982 a^{10} c+$ 


$$
\begin{aligned}
& +4788 a^{11} c-14 a^{12} c+234198965910666240 c^{2}+176184125195674752 a c^{2} \\
& +34997280444483552 a^{2} c^{2}+ \\
& +1019255391215056 a^{3} c^{2}-178004305027800 a^{4} c^{2}-3137600392428 a^{5} c^{2} \\
& +384865270776 a^{6} c^{2}-2779008876 a^{7} c^{2}- \\
& -186739560 a^{8} c^{2}+3261020 a^{9} c^{2}-6552 a^{10} c^{2}-84 a^{11} c^{2}-105436987989000192 c^{3} \\
& -57440774292713472 a c^{3}- \\
& -7587729681637376 a^{2} c^{3}+30167244537600 a^{3} c^{3}+31516664669440 a^{4} c^{3} \\
& -267758688960 a^{5} c^{3}-36622587456 a^{6} c^{3}+ \\
& +700882560 a^{7} c^{3}+3534720 a^{8} c^{3}-127680 a^{9} c^{3}+448 a^{10} c^{3}+29272395221864448 c^{4} \\
& +11567453274584576 a c^{4}+ \\
& +959504446627200 a^{2} c^{4}-30984268494080 a^{3} c^{4}-2804671306080 a^{4} c^{4} \\
& +71402698080 a^{5} c^{4}+1330539840 a^{6} c^{4}- \\
& -42060480 a^{7} c^{4}+151200 a^{8} c^{4}+1120 a^{9} c^{4}-5398575951060992 c^{5} \\
& -1531129739443200 a c^{5}-71188221133056 a^{2} c^{5}+ \\
& +4429295426304 a^{3} c^{5}+121707496512 a^{4} c^{5}-5277242880 a^{5} c^{5}+744576 a^{6} c^{5} \\
& +919296 a^{7} c^{5}-4032 a^{8} c^{5}+ \\
& +689572103331840 c^{6}+137397211710464 a c^{6}+2713645670400 a^{2} c^{6} \\
& -317715584256 a^{3} c^{6}-1414103040 a^{4} c^{6}+ \\
& +177612288 a^{5} c^{6}-1032192 a^{6} c^{6}-5376 a^{7} c^{6}-62363802566656 c^{7} \\
& -8441027076096 a c^{7}-5987837952 a^{2} c^{7}+ \\
& +12894704640 a^{3} c^{7}-76968960 a^{4} c^{7}-2626560 a^{5} c^{7}+15360 a^{6} c^{7}+4022644322304 c^{8} \\
& +351717355008 a c^{8}- \\
& -4223888640 a^{2} c^{8}-291152640 a^{3} c^{8}+2868480 a^{4} c^{8}+11520 a^{5} c^{8}-183833481216 c^{9} \\
& -9617372160 a c^{9}+ \\
& +185546240 a^{2} c^{9}+3210240 a^{3} c^{9}-28160 a^{4} c^{9}+5812561920 c^{1} 0+160635904 a c^{10} \\
& -3446784 a^{2} c^{10}-11264 a^{3} c^{10} \\
& \left.-120881152 c^{11}-1400832 a c^{11}+24576 a^{2} c^{11}+1486848 c^{12}+4096 a c^{12}-8192 c^{13}\right)+ \\
& +\frac{1}{\Gamma\left(\frac{c-a}{2}\right) \Gamma\left(\frac{c+a-27}{2}\right)}(-835776577757721600-732560266788865920 a \\
& -175421799959309568 a^{2}- \\
& -6354743845373448 a^{3}+1435874126626940 a^{4}+34847676651046 a^{5} \\
& -5714447085179 a^{6}+56439882581 a^{7}+
\end{aligned}
$$




$$
\begin{aligned}
& +6367545345 a^{8}-190140027 a^{9}+798847 a^{10}+31367 a^{11}-385 a^{12}+a^{13} \\
& +1296685506612695040 c+ \\
& +843440768583257088 a c+137263732473974976 a^{2} c-759650829872528 a^{3} c \\
& -970433188681304 a^{4} c+ \\
& +11103150302292 a^{5} c+2258084186290 a^{6} c-66672519396 a^{7} c-586759950 a^{8} c \\
& +43633100 a^{9} c-481306 a^{10} c+84 a^{11} c+ \\
& +14 a^{12} c-843760907368455168 c^{2}-407726081879932800 a c^{2} \\
& -42627997732439328 a^{2} c^{2}+1851674567870416 a^{3} c^{2}+ \\
& +226586093902440 a^{4} c^{2}-8357231234028 a^{5} c^{2}-240471792456 a^{6} c^{2} \\
& +13132553364 a^{7} c^{2}-99628200 a^{8} c^{2}- \\
& -2195620 a^{9} c^{2}+32424 a^{10} c^{2}-84 a^{11} c^{2}+310294269783343104 c^{3} \\
& +110429360845348864 a c^{3}+ \\
& +6630455726683136 a^{2} c^{3}-576173662357760 a^{3} c^{3}-22396834908160 a^{4} c^{3} \\
& +1518843466560 a^{5} c^{3}-733569984 a^{6} c^{3}- \\
& -930840960 a^{7} c^{3}+12835200 a^{8} c^{3}-2240 a^{9} c^{3}-448 a^{10} c^{3}-72831305901223936 c^{4} \\
& -18763381574731264 a c^{4}- \\
& -488099338700160 a^{2} c^{4}+85064353434880 a^{3} c^{4}+542806813920 a^{4} c^{4} \\
& -124938122400 a^{5} c^{4}+1528927680 a^{6} c^{4}+ \\
& +23419200 a^{7} c^{4}-433440 a^{8} c^{4}+1120 a^{9} c^{4}+11625428332593152 c^{5} \\
& +2111137037294592 a c^{5}+ \\
& +364443241728 a^{2} c^{5}-7095335831808 a^{3} c^{5}+75517299648 a^{4} c^{5}+5026549248 a^{5} c^{5} \\
& -92416128 a^{6} c^{5}+16128 a^{7} c^{5}+ \\
& +4032 a^{8} c^{5}-1307344350531584 c^{6}-160943873108992 a c^{6}+3096581440512 a^{2} c^{6} \\
& +347557964544 a^{3} c^{6}- \\
& -7182551040 a^{4} c^{6}-84306432 a^{5} c^{6}+2085888 a^{6} c^{6}-5376 a^{7} c^{6}+105454131576832 c^{7} \\
& +8307462070272 a c^{7}- \\
& -280139655168 a^{2} c^{7}-9574394880 a^{3} c^{7}+264053760 a^{4} c^{7}-46080 a^{5} c^{7}-15360 a^{6} c^{7} \\
& -6129210697728 c^{8}- \\
& -282331740672 a c^{8}+12658510080 a^{2} c^{8}+120433920 a^{3} c^{8}-4481280 a^{4} c^{8}+11520 a^{5} c^{8} \\
& +254489174016 c^{9}+ \\
& +5851028480 a c^{9}-322741760 a^{2} c^{9}+56320 a^{3} c^{9}+28160 a^{4} c^{9}-7360821248 c^{10} \\
& -58876928 a c^{10}+4392960 a^{2} c^{10}
\end{aligned}
$$




$$
\begin{aligned}
& -11264 a^{3} c^{10}+140836864 c^{11}-24576 a c^{11}-24576 a^{2} c^{11} \\
& \left.\left.-1601536 c^{12}+4096 a c^{12}+8192 c^{13}\right)\right] \\
& { }_{2} F_{1}\left[\begin{array}{ccc}
a, 29-a ; & \frac{1}{2} \\
c & ; & 2
\end{array}\right]= \\
& =\frac{\sqrt{\pi} \Gamma(c)}{2^{c-29}\left\{\prod_{\varphi=1}^{28}(\varphi-a)\right\}}\left[\frac{1}{\Gamma\left(\frac{c-a+1}{2}\right) \Gamma\left(\frac{c+a-28}{2}\right)}(3497296636753920000\right. \\
& +5555901589554355200 a+ \\
& +2361133612996790400 a^{2}+296837573062012416 a^{3}-8045509822184520 a^{4} \\
& -2309495184101812 a^{5} \\
& +52230988296494 a^{6}+5767788455233 a^{7}-229366815305 a^{8}-1161910491 a^{9} \\
& +180370407 a^{10}-2797949 a^{11}+ \\
& +3325 a^{12}+203 a^{13}-a^{14}-7918705667206656000 c-8468671081133975040 a c \\
& -2479748097907906560 a^{2} c- \\
& -171617279580731040 a^{3} c+14728842045867600 a^{4} c+1017261537926760 a^{5} c \\
& -60706566010020 a^{6} c- \\
& -943838942760 a^{7} c+96558281820 a^{8} c-1243943400 a^{9} c-18619020 a^{10} c+511560 a^{11} c \\
& -2940 a^{12} c+ \\
& +6846504051410380800 c^{2}+5236025170478554368 a c^{2}+1054763431379223552 a^{2} c^{2} \\
& +27669690834216368 a^{3} c^{2}- \\
& -6282770101240920 a^{4} c^{2}-97054310426892 a^{5} c^{2}+16257739742734 a^{6} c^{2} \\
& -161294509908 a^{7} c^{2}-10458065394 a^{8} c^{2}+ \\
& +246088780 a^{9} c^{2}-790566 a^{10} c^{2}-17052 a^{11} c^{2}+98 a^{1} 2 c^{2}-3194920175887319040 c^{3} \\
& -1785014716346695680 a c^{3}- \\
& -240816902070528000 a^{2} c^{3}+2045465086963200 a^{3} c^{3}+1188161185612800 a^{4} c^{3} \\
& -14134893897600 a^{5} c^{3}- \\
& -1735016492160 a^{6} c^{3}+42316243200 a^{7} c^{3}+228614400 a^{8} c^{3}-13641600 a^{9} c^{3} \\
& +94080 a^{10} c^{3}+928567869196713984 c^{4}+ \\
& +380255719502460928 a c^{4}+32418584463820800 a^{2} c^{4}-1288125293230720 a^{3} c^{4} \\
& -115836728362880 a^{4} c^{4}+ \\
& +3658531836960 a^{5} c^{4}+72650029536 a^{6} c^{4}-3160758720 a^{7} c^{4}+17357760 a^{8} c^{4} \\
& +227360 a^{9} c^{4}-1568 a^{10} c^{4}- \\
& -181307281284218880 c^{5}-53972606059223040 a c^{5}-2584272171962880 a^{2} c^{5} \\
& +195478551313920 a^{3} c^{5}+
\end{aligned}
$$


$+5608442125440 a^{4} c^{5}-313713146880 a^{5} c^{5}+282804480 a^{6} c^{5}+98219520 a^{7} c^{5}$ $-846720 a^{8} c^{5}+24848775724408832 c^{6}+$

$+5280766105517056 a c^{6}+104851642776832 a^{2} c^{6}-15801579295488 a^{3} c^{6}$ $-64686839616 a^{4} c^{6}+13307653632 a^{5} c^{6}-$

$-116038272 a^{6} c^{6}-1091328 a^{7} c^{6}+9408 a^{8} c^{6}-2451416728535040 c^{7}$ $-361346358804480 a c^{7}+301765201920 a^{2} c^{7}+$

$+759844915200 a^{3} c^{7}-6318950400 a^{4} c^{7}-280627200 a^{5} c^{7}+3225600 a^{6} c^{7}$ $+176187932065792 c^{8}+$

$+17257259464704 a c^{8}-269957270016 a^{2} c^{8}-21766536960 a^{3} c^{8}+318769920 a^{4} c^{8}$ $+2338560 a^{5} c^{8}-26880 a^{6} c^{8}-$

$-9232454246400 c^{9}-563530598400 a c^{9}+14458752000 a^{2} c^{9}+342988800 a^{3} c^{9}$ $-5913600 a^{4} c^{9}+348808728576 c^{10}+$

$+11988601856 a c^{10}-380244480 a^{2} c^{10}-2286592 a^{3} c^{10}+39424 a^{4} c^{10}-9248440320 c^{11}$ $-149667840 a c^{11}$

$\left.+5160960 a^{2} c^{11}+163201024 c^{12}+831488 a c^{12}-28672 a^{2} c^{12}-1720320 c^{13}+8192 c^{14}\right)+$ $+\frac{1}{\Gamma\left(\frac{c-a}{2}\right) \Gamma\left(\frac{c+a-29}{2}\right)}(1613913397544448000+1472574330511280640 a$ $+389021279417326080 a^{2}+$

$+24196510822078560 a^{3}-2274657906963120 a^{4}-143258950036440 a^{5}$ $+8920185210300 a^{6}+128774925720 a^{7}-$

$-13826578500 a^{8}+179655000 a^{9}+2646420 a^{10}-73080 a^{11}+420 a^{12}$ $-2508255032493772800 c-$

$-1717842284795966976 a c-319975449572777472 a^{2} c-7155985266367904 a^{3} c$ $+1861324669488208 a^{4} c+$

$+25627804125096 a^{5} c-4686483308420 a^{6} c+47891228952 a^{7} c+2978091900 a^{8} c$ $-70441000 a^{9} c+226772 a^{10} c+$

$+4872 a^{11} c-28 a^{12} c+1635663709850664960 c^{2}+843196968357212160 a c^{2}$ $+106898081437578240 a^{2} c^{2}-$

$-1158164266406400 a^{3} c^{2}-517205009606400 a^{4} c^{2}+6506815190400 a^{5} c^{2}$ $+743164813440 a^{6} c^{2}-18275846400 a^{7} c^{2}-$

$-96768000 a^{8} c^{2}+5846400 a^{9} c^{2}-40320 a^{10} c^{2}-603022201203621888 c^{3}$ $-232515149352194048 a c^{3}-$ 


$$
\begin{aligned}
& -18822315352579072 a^{2} c^{3}+781393137041920 a^{3} c^{3}+66374684913920 a^{4} c^{3} \\
& -2128630197120 a^{5} c^{3}- \\
& -41182549632 a^{6} c^{3}+1809265920 a^{7} c^{3}-9945600 a^{8} c^{3}-129920 a^{9} c^{3}+896 a^{10} c^{3} \\
& +141928904910028800 c^{4}+ \\
& +40368743871436800 a c^{4}+1844139367411200 a^{2} c^{4}-143432935027200 a^{3} c^{4} \\
& -3974328892800 a^{4} c^{4}+ \\
& +225483955200 a^{5} c^{4}-218131200 a^{6} c^{4}-70156800 a^{7} c^{4}+604800 a^{8} c^{4} \\
& -22721029659049984 c^{5}- \\
& -4664860707047424 a c^{5}-87699486460416 a^{2} c^{5}+13718425437696 a^{3} c^{5} \\
& +52894283904 a^{4} c^{5}-11425268736 a^{5} c^{5}+ \\
& +99676416 a^{6} c^{5}+935424 a^{7} c^{5}-8064 a^{8} c^{5}+2562816998277120 c^{6} \\
& +368118604062720 a c^{6}-475610849280 a^{2} c^{6}- \\
& -763960780800 a^{3} c^{6}+6389913600 a^{4} c^{6}+280627200 a^{5} c^{6}-3225600 a^{6} c^{6} \\
& -207355683897344 c^{7}-19928195211264 a c^{7}+ \\
& +315044315136 a^{2} c^{7}+24915240960 a^{3} c^{7}-364984320 a^{4} c^{7}-2672640 a^{5} c^{7}+30720 a^{6} c^{7} \\
& +12088613560320 c^{8}+ \\
& +728067225600 a c^{8}-18711475200 a^{2} c^{8}-440985600 a^{3} c^{8}+7603200 a^{4} c^{8} \\
& -503429701632 c^{9}-17152706560 a c^{9}+ \\
& +544107520 a^{2} c^{9}+3266560 a^{3} c^{9}-56320 a^{4} c^{9}+14603550720 c^{10}+235192320 a c^{10} \\
& -8110080 a^{2} c^{10}-280199168 c^{11}- \\
& \left.\left.-1425408 a c^{11}+49152 a^{2} c^{11}+3194880 c^{12}-16384 c^{13}\right)\right] \\
& { }_{2} F_{1}\left[\begin{array}{ccc}
a, 30-a ; & \frac{1}{2} \\
c & ; & =
\end{array}\right]= \\
& =\frac{\sqrt{\pi} \Gamma(c)}{2^{c-30}\left\{\prod_{\mathcal{X}=1}^{29}(\varkappa-a)\right\}}\left[\frac{1}{\Gamma\left(\frac{c-a+1}{2}\right) \Gamma\left(\frac{c+a-30}{2}\right)}(-6761440164390912000\right. \\
& -10975820682116828160 a- \\
& -4935233255279884416 a^{2}-729789149421755136 a^{3}-4220197814149688 a^{4} \\
& +4751293608022388 a^{5}+ \\
& +20580722419698 a^{6}-12993499610273 a^{7}+207448509161 a^{8}+9479314299 a^{9} \\
& -309595783 a^{10}+1647709 a^{11}+ \\
& +35427 a^{12}-427 a^{13}+a^{14}+15326298997068810240 c+16912364017713907968 a c \\
& +5341563944575762176 a^{2} c+
\end{aligned}
$$


$+486193174327051888 a^{3} c-17721927335789288 a^{4} c-2655392142674852 a^{5} c$ $+68415639214898 a^{6} c+$

$+4076609731294 a^{7} c-151651399494 a^{8} c-156424450 a^{9} c+60914966 a^{10} c$ $-708022 a^{11} c+742 a^{12} c+14 a^{13} c-$

$-13273287874275557376 c^{2}-10581609146505394176 a c^{2}$ $-2359216299514810368 a^{2} c^{2}-113984108675034240 a^{3} c^{2}+$

$+10464620334250304 a^{4} c^{2}+462806131944480 a^{5} c^{2}-27019467604880 a^{6} c^{2}$ $-213739449504 a^{7} c^{2}+24126311664 a^{8} c^{2}-$

$-258857760 a^{9} c^{2}-2150960 a^{10} c^{2}+40992 a^{11} c^{2}-112 a^{12} c^{2}+6206845674605838336 c^{3}$ $+3655758996318896128 a c^{3}+$

$+566360717498711040 a^{2} c^{3}+8471349113362176 a^{3} c^{3}-2354033956865280 a^{4} c^{3}$ $-18437749885120 a^{5} c^{3}+$

$+3762081665664 a^{6} c^{3}-35808999744 a^{7} c^{3}-1303626240 a^{8} c^{3}+23047360 a^{9} c^{3}$ $-51072 a^{10} c^{3}-448 a^{11} c^{3}-$

$-1808232623781232640 c^{4}-790682156498434048 a c^{4}-82059994215443456 a^{2} c^{4}$ $+1023013859383680 a^{3} c^{4}+$

$+271425411296640 a^{4} c^{4}-3312431939040 a^{5} c^{4}-235347504672 a^{6} c^{4}+4630785600 a^{7} c^{4}$ $+13513920 a^{8} c^{4}-$

$-614880 a^{9} c^{4}+2016 a^{10} c^{4}+353978861595516928 c^{5}+114221183100068864 a c^{5}$ $+7421904568692480 a^{2} c^{5}-$

$-278541991695360 a^{3} c^{5}-17101748153280 a^{4} c^{5}+460523722176 a^{5} c^{5}$ $+5856950400 a^{6} c^{5}-195941760 a^{7} c^{5}+$

$+705600 a^{8} c^{5}+4032 a^{9} c^{5}-48646217148710912 c^{6}-11412178770864128 a c^{6}$ $-406527582805504 a^{2} c^{6}+$

$+27328469113344 a^{3} c^{6}+538828983168 a^{4} c^{6}-24621112320 a^{5} c^{6}+27928320 a^{6} c^{6}$ $+3279360 a^{7} c^{6}-13440 a^{8} c^{6}+$

$+4812572103507968 c^{7}+801309941284864 a c^{7}+11028903438336 a^{2} c^{7}$ $-1492276712448 a^{3} c^{7}-3166279680 a^{4} c^{7}+$

$+645550080 a^{5} c^{7}-3624960 a^{6} c^{7}-15360 a^{7} c^{7}-346867926056960 c^{8}$ $-39565589673984 a c^{8}+77974516224 a^{2} c^{8}+$

$+48272843520 a^{3} c^{8}-301551360 a^{4} c^{8}-7729920 a^{5} c^{8}+42240 a^{6} c^{8}+18227487657984 c^{9}$ $+1352747703296 a c^{9}-$ 


$$
\begin{aligned}
& -16971947520 a^{2} c^{9}-893770240 a^{3} c^{9}+8363520 a^{4} c^{9}+28160 a^{5} c^{9}-690554748928 c^{10} \\
& -30856196096 a c^{10}+ \\
& +573089792 a^{2} c^{10}+8245248 a^{3} c^{10}-67584 a^{4} c^{10}+18358992896 c^{11}+435724288 a c^{11} \\
& -8798208 a^{2} c^{11}- \\
& -24576 a^{3} c^{11}-324812800 c^{12}-3248128 a c^{12}+53248 a^{2} c^{12}+3432448 c^{13}+8192 a c^{13} \\
& \left.-16384 c^{14}\right)+ \\
& +\frac{c}{\Gamma\left(\frac{c-a}{2}\right) \Gamma\left(\frac{c+a-29}{2}\right)}(46803488615967283200+42767159087365735680 a \\
& +11270601192070601856 a^{2}+ \\
& +336434811648432 a^{6}+ \\
& +661015913631944304 a^{3}-76584910384046512 a^{4}-4417245600019672 a^{5} \\
& +3976287855967 a^{7}-608379703391 a^{8}+9950104899 a^{9}+147972013 a^{10}-5745971 a^{11} \\
& +49203 a^{12}-7 a^{13}-a^{14}- \\
& +4413443650 a^{9} c+20549494 a^{10} c+541898 a^{11} c-6202 a^{12} c+14 a^{13} c \\
& +50034321004680953856 c^{2}+
\end{aligned}
$$

$+26188677313214367744 a c^{2}+3340987169121850368 a^{2} c^{2}-55647356127077760 a^{3} c^{2}$ $-18708842259562304 a^{4} c^{2}+$$$
+314035097808480 a^{5} c^{2}+32131358547920 a^{6} c^{2}-977872074144 a^{7} c^{2}
$$$$
-4833796464 a^{8} c^{2}+459678240 a^{9} c^{2}-
$$$$
-4723600 a^{10} c^{2}+672 a^{11} c^{2}+112 a^{12} c^{2}-19178889908274610176 c^{3}
$$$$
-7572855930087606272 a c^{3}-
$$

$-617930250420794880 a^{2} c^{3}+31207762807608576 a^{3} c^{3}+2572172951249280 a^{4} c^{3}$$$
-100552461707200 a^{5} c^{3}-
$$$$
-1945358951424 a^{6} c^{3}+112831486656 a^{7} c^{3}-854904960 a^{8} c^{3}-14450240 a^{9} c^{3}
$$$$
+198912 a^{10} c^{3}-448 a^{11} c^{3}+
$$$$
+4737652999555604480 c^{4}+1394083224658414592 a c^{4}+63770234054656256 a^{2} c^{4}
$$$$
-5994963943259520 a^{3} c^{4}-
$$

$-165690819368640 a^{4} c^{4}+11870436900000 a^{5} c^{4}-26042015328 a^{6} c^{4}-5516145600 a^{7} c^{4}$ $+70855680 a^{8} c^{4}-$ 


$$
\begin{aligned}
& -10080 a^{9} c^{4}-2016 a^{10} c^{4}-804781701766094848 c^{5}-173082746257294336 a c^{5} \\
& -3095138947918080 a^{2} c^{5}+ \\
& +628850081495040 a^{3} c^{5}+1766625026880 a^{4} c^{5}-710151349824 a^{5} c^{5}+8365123200 a^{6} c^{5} \\
& +104039040 a^{7} c^{5}- \\
& -1794240 a^{8} c^{5}+4032 a^{9} c^{5}+97597927269220352 c^{6}+14916118888978432 a c^{6} \\
& -53460036102656 a^{2} c^{6}- \\
& -39840066986496 a^{3} c^{6}+440358344832 a^{4} c^{6}+22064609280 a^{5} c^{6}-377905920 a^{6} c^{6} \\
& +53760 a^{7} c^{6}+13440 a^{8} c^{6}- \\
& -8630073563660288 c^{7}-900484605464576 a c^{7}+17956861353984 a^{2} c^{7} \\
& +1544786045952 a^{3} c^{7}-30214072320 a^{4} c^{7}- \\
& -297246720 a^{5} c^{7}+6850560 a^{6} c^{7}-15360 a^{7} c^{7}+561592474234880 c^{8} \\
& +37734427923456 a c^{8}-1220290689024 a^{2} c^{8}- \\
& -34672999680 a^{3} c^{8}+890799360 a^{4} c^{8}-126720 a^{5} c^{8}-42240 a^{6} c^{8}-26862108708864 c^{9} \\
& -1061440635904 a c^{9}+ \\
& +44645061120 a^{2} c^{9}+363292160 a^{3} c^{9}-12587520 a^{4} c^{9}+28160 a^{5} c^{9}+932581163008 c^{10} \\
& +18492289024 a c^{10}- \\
& -950208512 a^{2} c^{10}+135168 a^{3} c^{10}+67584 a^{4} c^{10}-22848782336 c^{11}-158523392 a c^{11} \\
& +11010048 a^{2} c^{11}- \\
& -24576 a^{3} c^{11}+374333440 c^{12}-53248 a c^{12}-53248 a^{2} c^{12} \\
& \left.\left.-3678208 c^{13}+8192 a c^{13}+16384 c^{14}\right)\right] \\
& { }_{2} F_{1}\left[\begin{array}{ccc}
a, 31-a ; & \frac{1}{2} \\
c & ; & 2
\end{array}\right]= \\
& =\frac{\sqrt{\pi} \Gamma(c)}{2^{c-31}\left\{\prod_{\vartheta=1}^{30}(\vartheta-a)\right\}}\left[\frac{1}{\Gamma\left(\frac{c-a+1}{2}\right) \Gamma\left(\frac{c+a-30}{2}\right)}(-202843204931727360000\right. \\
& -329526641070543974400 a- \\
& -148192702618125404160 a^{2}-21658122462897527040 a^{3}+9722889879655680 a^{4} \\
& +159098559658389120 a^{5}- \\
& -967254435840 a^{6}-490253327976720 a^{7}+9909256423440 a^{8}+414792045360 a^{9} \\
& -17018472240 a^{10}+ \\
& +122231760 a^{11}+2840880 a^{12}-52080 a^{13}+240 a^{14}+466802430683494348800 c \\
& +519033146796495256320 a c+
\end{aligned}
$$


$+164949222985279959168 a^{2} c+14769765039630034320 a^{3} c$

$+2935763232708224 a^{6} c+161360534973585 a^{7} c-7364342102145 a^{8} c$ $+2500344525 a^{9} c+3911185523 a^{10} c-$

$-60969405 a^{11} c+109165 a^{12} c+3255 a^{13} c-15 a^{14} c-414075635848567848960 c^{2}$ $-334813261843713392640 a c^{2}-$

$$
-75572573936717905920 a^{2} c^{2}-3508604162375731200 a^{3} c^{2}
$$

$+392743927281884160 a^{4} c^{2}+$

$+16485242844787200 a^{5} c^{2}-1178569050412800 a^{6} c^{2}-7610697884160 a^{7} c^{2}$ $+1309085487360 a^{8} c^{2}-$

$-18023846400 a^{9} c^{2}-167865600 a^{10} c^{2}+4999680 a^{11} c^{2}-26880 a^{12} c^{2}$

$+199928658130806030336 c^{3}+$

$+120313411708083025920 a c^{3}+18991014146939294720 a^{2} c^{3}$

$+228969490870081920 a^{3} c^{3}-$

$-92456288210742080 a^{4} c^{3}-536544028552800 a^{5} c^{3}+178570308069840 a^{6} c^{3}$ $-2197559659680 a^{7} c^{3}-$

$-82666918320 a^{8} c^{3}+1975394400 a^{9} c^{3}-6824720 a^{10} c^{3}-104160 a^{11} c^{3}+560 a^{12} c^{3}$ $-60648845538056601600 c^{4}-$

$-27329375418592542720 a c^{4}-2908495070746890240 a^{2} c^{4}+49850050806835200 a^{3} c^{4}$ $+$

$+11474023776537600 a^{4} c^{4}-180112947033600 a^{5} c^{4}-12591968417280 a^{6} c^{4}$

$+315229824000 a^{7} c^{4}+945100800 a^{8} c^{4}-$

$-74995200 a^{9} c^{4}+483840 a^{1} 0 c^{4}+12479370238337146880 c^{5}$

$+4192758239972766720 a c^{5}+$

$+280853229778835712 a^{2} c^{5}-12726157894300800 a^{3} c^{5}-795295816175040 a^{4} c^{5}$

$+26431624205280 a^{5} c^{5}+$

$+360301027296 a^{6} c^{5}-16739553600 a^{7} c^{5}+91405440 a^{8} c^{5}+937440 a^{9} c^{5}-6048 a^{10} c^{5}$

$-1822444137279651840 c^{6}-$

$-450829569304657920 a c^{6}-16543535489064960 a^{2} c^{6}+1351753242255360 a^{3} c^{6}$ $+27946244229120 a^{4} c^{6}-$

$-1656693964800 a^{5} c^{6}+3348172800 a^{6} c^{6}+399974400 a^{7} c^{6}-3225600 a^{8} c^{6}$

$+194120876679053312 c^{7}+$ 


$$
\begin{aligned}
& +34630926348380160 a c^{7}+471615845905920 a^{2} c^{7}-83776087272960 a^{3} c^{7} \\
& -132129333120 a^{4} c^{7}+ \\
& +55017907200 a^{5} c^{7}-462432000 a^{6} c^{7}-3571200 a^{7} c^{7}+28800 a^{8} c^{7} \\
& -15312178746163200 c^{8}-1910620958146560 a c^{8}+ \\
& +6959519170560 a^{2} c^{8}+3225307852800 a^{3} c^{8}-27665510400 a^{4} c^{8}-942796800 a^{5} c^{8} \\
& +10137600 a^{6} c^{8}+ \\
& +899363697520640 c^{9}+75101815557120 a c^{9}-1211625451520 a^{2} c^{9} \\
& -76032633600 a^{3} c^{9}+1057196800 a^{4} c^{9}+ \\
& +6547200 a^{5} c^{9}-70400 a^{6} c^{9}-39186998231040 c^{10}-2051861053440 a c^{10} \\
& +50601492480 a^{2} c^{10}+ \\
& +1005649920 a^{3} c^{10}-16220160 a^{4} c^{10}+1248504659968 c^{11}+37012869120 a c^{11} \\
& -1105397760 a^{2} c^{11}-5713920 a^{3} c^{11}+ \\
& +92160 a^{4} c^{11}-28242739200 c^{12}-396165120 a c^{12}+12779520 a^{2} c^{12}+429383680 c^{13} \\
& +1904640 a c^{13}- \\
& \left.-61440 a^{2} c^{13}-3932160 c^{14}+16384 c^{15}\right)+\frac{1}{\Gamma\left(\frac{c-a}{2}\right) \Gamma\left(\frac{c+a-31}{2}\right)}(-90587397405593088000 \\
& -85809869707963968000 a- \\
& -24562874768681744640 a^{2}-1998123269397359328 a^{3}+103194363041532000 a^{4} \\
& +12301377002464816 a^{5}- \\
& -415292450573728 a^{6}-21219497625214 a^{7}+992922344830 a^{8}-596890182 a^{9} \\
& -520580634 a^{10}+8143142 a^{11}- \\
& -14630 a^{12}-434 a^{1} 3+2 a^{14}+144240561108261273600 c+104221907769153945600 a c \\
& + \\
& +21717111091604815872 a^{2} c+907956238606835712 a^{3} c-110873520753201152 a^{4} c \\
& -4298678159370240 a^{5} c+ \\
& +320998107247616 a^{6} c+1861076883456 a^{7} c-349590936576 a^{8} c+4846356480 a^{9} c \\
& +44506112 a^{10} c-1333248 a^{11} c+ \\
& +7168 a^{12} c-97158488130721382400 c^{2}-53813036357134553088 a c^{2} \\
& -7972191332273645568 a^{2} c^{2}- \\
& +901353268032 a^{7} c^{2}+32944812768 a^{8} c^{2}-791407680 a^{9} c^{2}+2737952 a^{10} c^{2} \\
& +41664 a^{11} c^{2}-224 a^{12} c^{2}+ \\
& +37320860741630164992 c^{3}+15792177885946970112 a c^{3} \\
& +1595260691293962240 a^{2} c^{3}-
\end{aligned}
$$




$$
\begin{aligned}
& -30215467465359360 a^{3} c^{3}-6193795836764160 a^{4} c^{3}+100485128540160 a^{5} c^{3} \\
& +6706712334336 a^{6} c^{3}- \\
& -169189171200 a^{7} c^{3}-495452160 a^{8} c^{3}+39997440 a^{9} c^{3}-258048 a^{10} c^{3} \\
& -9240641868654624768 c^{4}- \\
& -2958046488856795136 a c^{4}-189415956515840512 a^{2} c^{4}+8876337936464640 a^{3} c^{4} \\
& +530796049376640 a^{4} c^{4}- \\
& -17877943030080 a^{5} c^{4}-238041486144 a^{6} c^{4}+11176368000 a^{7} c^{4}-61071360 a^{8} c^{4} \\
& -624960 a^{9} c^{4}+4032 a^{10} c^{4}+ \\
& +1573605911085187072 c^{5}+374998609630986240 a c^{5}+13181162421780480 a^{2} c^{5} \\
& -1105518682275840 a^{3} c^{5}- \\
& -22149881241600 a^{4} c^{5}+1332394721280 a^{5} c^{5}-2754232320 a^{6} c^{5}-319979520 a^{7} c^{5} \\
& +2580480 a^{8} c^{5}- \\
& -191329364453654528 c^{6}-33165618687913984 a c^{6}-426536477895680 a^{2} c^{6} \\
& +79042932261888 a^{3} c^{6}+ \\
& +111474429696 a^{4} c^{6}-51423375360 a^{5} c^{6}+432391680 a^{6} c^{6}+3333120 a^{7} c^{6}-26880 a^{8} c^{6} \\
& +16962966866886656 c^{7}+ \\
& +2070867300581376 a c^{7}-8234460512256 a^{2} c^{7}-3456418775040 a^{3} c^{7} \\
& +29769400320 a^{4} c^{7}+1005649920 a^{5} c^{7}- \\
& -10813440 a^{6} c^{7}-1106777235841024 c^{8}-90936788625408 a c^{8}+1478279835648 a^{2} c^{8} \\
& +91364866560 a^{3} c^{8}- \\
& -1270663680 a^{4} c^{8}-7856640 a^{5} c^{8}+84480 a^{6} c^{8}+53078834282496 c^{9} \\
& +2747435581440 a c^{9}-67843522560 a^{2} c^{9}- \\
& -1340866560 a^{3} c^{9}+21626880 a^{4} c^{9}-1847520559104 c^{10}-54358171648 a c^{10} \\
& +1623592960 a^{2} c^{10}+8380416 a^{3} c^{10}- \\
& -135168 a^{4} c^{10}+45379223552 c^{11}+633864192 a c^{11}-20447232 a^{2} c^{11}-745259008 c^{12} \\
& -3301376 a c^{12}+ \\
& \left.\left.+106496 a^{2} c^{12}+7340032 c^{13}-32768 c^{14}\right)\right]
\end{aligned}
$$

\section{MAIN SUMMATION FORMULAE}

$$
{ }_{2} F_{1}\left[\begin{array}{ccc}
a, & 32-a ; & \frac{1}{2} \\
c & ; & 2
\end{array}\right]=
$$




$$
\begin{gathered}
=\frac{\sqrt{\pi} \Gamma(c)}{2^{c-32}\left\{\prod_{\varpi=1}^{31}(\varpi-a)\right\}}\left[\frac{1}{\Gamma\left(\frac{c-a+1}{2}\right) \Gamma\left(\frac{c+a-32}{2}\right)}(393008709555221760000\right. \\
+651194829215986003200 a+
\end{gathered}
$$

$$
\begin{gathered}
+307720612018593866880 a^{2}+51153440461235528688 a^{3}+1330940239653764544 a^{4} \\
-296857314253591112 a^{5}-
\end{gathered}
$$$$
-7921227385233624 a^{6}+908822265956143 a^{7}+402064837152 a^{8}-1099599340796 a^{9}
$$$$
+20437153968 a^{10}+
$$$$
+154052570 a^{11}-7588896 a^{12}+63308 a^{13}-24 a^{14}-a^{15}-905281327552108953600 c
$$$$
-1035422190413513441280 a c-
$$$$
-351184084010029910016 a^{2} c-38338797951234352128 a^{3} c
$$$$
+451749423064008064 a^{4} c+206106543083095360 a^{5} c
$$$$
-1447679061168672 a^{6} c-415046253507664 a^{7} c+8508403087952 a^{8} c
$$$$
+199440969840 a^{9} c-7487180848 a^{10} c+
$$

$$
\begin{gathered}
+47316752 a^{11} c+584304 a^{12} c-7280 a^{13} c+16 a^{14} c+804169092960729907200 c^{2} \\
+674782022139214399488 a c^{2}+ \\
+165841199803790742528 a^{2} c^{2}+10946190747818876544 a^{3} c^{2} \\
-567565724112283456 a^{4} c^{2}-
\end{gathered}
$$

$-48517995647477984 a^{5} c^{2}+1590023100897232 a^{6} c^{2}+53334001088048 a^{7} c^{2}$

$$
-2274073929456 a^{8} c^{2}+
$$$$
+4737986736 a^{9} c^{2}+642792304 a^{10} c^{2}-7496944 a^{11} c^{2}+9968 a^{12} c^{2}+112 a^{13} c^{2}
$$$$
-388961945977802194944 c^{3}-
$$

$$
-245240098536550637568 a c^{3}-43311426401359084544 a^{2} c^{3}
$$

$$
-1395255045945941760 a^{3} c^{3}+
$$

$$
\begin{gathered}
+165983593526283904 a^{4} c^{3}+4591339102100160 a^{5} c^{3}-322284615941984 a^{6} c^{3} \\
-765397859520 a^{7} c^{3}+ \\
+208379147424 a^{8} c^{3}-2341805760 a^{9} c^{3}-12518688 a^{10} c^{3}+262080 a^{11} c^{3}-672 a^{12} c^{3} \\
+118230509217761009664 c^{4}+ \\
+56420416163828755456 a c^{4}+6990463455382573824 a^{2} c^{4}+33709473394238336 a^{3} c^{4} \\
-23923420575560640 a^{4} c^{4}- \\
-35063040521760 a^{5} c^{4}+29185328399808 a^{6} c^{4}-321523472928 a^{7} c^{4}-7433455680 a^{8} c^{4} \\
+133744800 a^{9} c^{4}- \\
-310464 a^{10} c^{4}-2016 a^{11} c^{4}-24381290438326222848 c^{5}-8782568098611445760 a c^{5} \\
-
\end{gathered}
$$




$$
\begin{aligned}
& +13675686801784320 a^{3} c^{5}+1956221896719360 a^{4} c^{5}-28876551392640 a^{5} c^{5} \\
& -1297457659008 a^{6} c^{5}+ \\
& +26112871680 a^{7} c^{5}+40024320 a^{8} c^{5}-2620800 a^{9} c^{5}+8064 a^{10} c^{5} \\
& +3568887719037157376 c^{6}+ \\
& +960485441528322048 a c^{6}+49667323801539072 a^{2} c^{6}-2072805728685056 a^{3} c^{6} \\
& -91894140143232 a^{4} c^{6}+ \\
& +2584449716352 a^{5} c^{6}+22947859200 a^{6} c^{6}-826855680 a^{7} c^{6}+2943360 a^{8} c^{6} \\
& +13440 a^{9} c^{6}-381067609873448960 c^{7}- \\
& -75290787841798144 a c^{7}-2072992543587328 a^{2} c^{7}+150201995412480 a^{3} c^{7} \\
& +2162904361728 a^{4} c^{7}- \\
& -105258362880 a^{5} c^{7}+189150720 a^{6} c^{7}+10982400 a^{7} c^{7}-42240 a^{8} c^{7} \\
& +30132576016863232 c^{8}+4259476607648768 a c^{8}+ \\
& +39713460593664 a^{2} c^{8}-6478199887104 a^{3} c^{8}-3138938880 a^{4} c^{8}+2216670720 a^{5} c^{8} \\
& -11996160 a^{6} c^{8}-42240 a^{7} c^{8}- \\
& -1774218876289024 c^{9}-172982726737920 a c^{9}+673615990784 a^{2} c^{9} \\
& +171263600640 a^{3} c^{9}-1092044800 a^{4} c^{9}- \\
& -21964800 a^{5} c^{9}+112640 a^{6} c^{9}+77495587749888 c^{10}+4944943984640 a c^{10} \\
& -63368718336 a^{2} c^{10}- \\
& -2649946112 a^{3} c^{10}+23586816 a^{4} c^{10}+67584 a^{5} c^{10}-2474963632128 c^{11} \\
& -95577284608 a c^{11}+1707290624 a^{2} c^{11}+ \\
& +20766720 a^{3} c^{11}-159744 a^{4} c^{11}+56117960704 c^{12}+1156386816 a c^{12}-22044672 a^{2} c^{12} \\
& -53248 a^{3} c^{12}- \\
& \left.-855113728 c^{13}-7454720 a c^{13}+114688 a^{2} c^{13}+7847936 c^{14}+16384 a c^{14}-32768 c^{15}\right) \\
& +\frac{1}{\Gamma\left(\frac{c-a}{2}\right) \Gamma\left(\frac{c+a-31}{2}\right)}(-2808209320881060096000-2663360260009726636800 a \\
& -761012930360984837760 a^{2}- \\
& -59789981041407736848 a^{3}+3838097925675257664 a^{4}+412887209956538296 a^{5} \\
& -17102186004670536 a^{6}- \\
& -788560364968145 a^{7}+45162707702112 a^{8}-113794887932 a^{9}-29121053808 a^{10} \\
& +578885594 a^{11}-1601376 a^{12}- \\
& -54964 a^{13}+504 a^{14}-a^{15}+4565299095163699200000 c \\
& +3321179752803522232320 a c+
\end{aligned}
$$




$$
\begin{aligned}
& +693852416121601198080 a^{2} c+26869933301958220800 a^{3} c \\
& -4073155305339587968 a^{4} c- \\
& -144960223788782272 a^{5} c+13373713249687584 a^{6} c+48530135178160 a^{7} c \\
& -17254272251984 a^{8} c+ \\
& +297414505584 a^{9} c+2773686832 a^{10} c-118937840 a^{11} c+953232 a^{12} c-112 a^{13} c \\
& -16 a^{14} c- \\
& -3161080629046376939520 c^{2}-1773743611853439203328 a c^{2} \\
& -264570033924753638400 a^{2} c^{2}- \\
& -1405058881009041792 a^{3} c^{2}+1448652525436875584 a^{4} c^{2}+2719092197913376 a^{5} c^{2} \\
& -3193999982297552 a^{6} c^{2}+ \\
& +50491558757936 a^{7} c^{2}+1813029642480 a^{8} c^{2}-55967482704 a^{9} c^{2}+272830096 a^{10} c^{2} \\
& +5276432 a^{11} c^{2}-56560 a^{12} c^{2}+ \\
& +112 a^{13} c^{2}+1257211815368285749248 c^{3}+542821659317684920320 a c^{3} \\
& +55394584008959679488 a^{2} c^{3}- \\
& -1371732146004729600 a^{3} c^{3}-249881887807080064 a^{4} c^{3}+5098386147168960 a^{5} c^{3} \\
& +325475917621088 a^{6} c^{3}- \\
& -10145058512064 a^{7} c^{3}-25272802464 a^{8} c^{3}+3568138560 a^{9} c^{3}-34317024 a^{10} c^{3} \\
& +4032 a^{11} c^{3}+672 a^{12} c^{3}- \\
& -324881117647402082304 c^{4}-107015854008936485888 a c^{4} \\
& -6926032383442477824 a^{2} c^{4}+ \\
& +388761985674363776 a^{3} c^{4}+23023713558513600 a^{4} c^{4}-940801602446880 a^{5} c^{4} \\
& -12549395769792 a^{6} c^{4}+ \\
& +787489416672 a^{7} c^{4}-5878918080 a^{8} c^{4}-79144800 a^{9} c^{4}+1020096 a^{1} 0 c^{4}-2016 a^{1} 1 c^{4} \\
& +58269865681132978176 c^{5}+ \\
& +14434175239659732992 a c^{5}+507569977317398528 a^{2} c^{5}-51503477691302400 a^{3} c^{5} \\
& -1031098175523840 a^{4} c^{5}+ \\
& +78913013289600 a^{5} c^{5}-261319992192 a^{6} c^{5}-28545135360 a^{7} c^{5}+343176960 a^{8} c^{5} \\
& -40320 a^{9} c^{5}-8064 a^{10} c^{5}- \\
& -7542483540103512064 c^{6}-1375984874765199360 a c^{6}-16369409928073728 a^{2} c^{6} \\
& +4065809536857088 a^{3} c^{6}+ \\
& +1341327292032 a^{4} c^{6}-3613476171648 a^{5} c^{6}+40882033920 a^{6} c^{6}+422096640 a^{7} c^{6} \\
& -6814080 a^{8} c^{6}+13440 a^{9} c^{6}+
\end{aligned}
$$




$$
\begin{aligned}
& +721205116854075392 c^{7}+94113516697784320 a c^{7}-588042790421504 a^{2} c^{7} \\
& -203145224764416 a^{3} c^{7}+ \\
& +2278013244672 a^{4} c^{7}+89713367040 a^{5} c^{7}-1438103040 a^{6} c^{7}+168960 a^{7} c^{7} \\
& +42240 a^{8} c^{7}-51580334106447872 c^{8}- \\
& -4622210372353024 a c^{8}+93568112901120 a^{2} c^{8}+6406705933056 a^{3} c^{8} \\
& -118823147520 a^{4} c^{8}-994920960 a^{5} c^{8}+ \\
& +21457920 a^{6} c^{8}-42240 a^{7} c^{8}+2769079768711168 c^{9}+160632598511616 a c^{9} \\
& -4979646746624 a^{2} c^{9}- \\
& -119617935360 a^{3} c^{9}+2876262400 a^{4} c^{9}-337920 a^{5} c^{9}-112640 a^{6} c^{9} \\
& -111011105390592 c^{10}-3805382496256 a c^{10}+ \\
& +150700222464 a^{2} c^{10}+1061226496 a^{3} c^{10}-34400256 a^{4} c^{10}+67584 a^{5} c^{10} \\
& +3272190984192 c^{11}+56546713600 a c^{11}- \\
& -2719428608 a^{2} c^{11}+319488 a^{3} c^{11}+159744 a^{4} c^{11}-68803764224 c^{12}-418050048 a c^{12} \\
& +27156480 a^{2} c^{12} \\
& -53248 a^{3} c^{12}+976224256 c^{13}-114688 a c^{13}-114688 a^{2} c^{13} \\
& \left.\left.-8372224 c^{14}+16384 a c^{14}+32768 c^{15}\right)\right] \\
& { }_{2} F_{1}\left[\begin{array}{ccc}
a, & 33-a ; & \frac{1}{2} \\
c & ; & =
\end{array}=\right. \\
& =\frac{\sqrt{\pi} \Gamma(c)}{2^{c-33}\left\{\prod_{\tau=1}^{32}(\tau-a)\right\}}\left[\frac{1}{\Gamma\left(\frac{c-a+1}{2}\right) \Gamma\left(\frac{c+a-32}{2}\right)}(12576278705767096320000\right. \\
& +20851860424556970547200 a+ \\
& +9854947407585176052480 a^{2}+1624435436389013576064 a^{3} \\
& +34691223091312022544 a^{4}- \\
& -10657758864295730880 a^{5}-242490144783523000 a^{6}+36347810238286008 a^{7} \\
& -92082876669487 a^{8}- \\
& -50233536212640 a^{9}+1138218046460 a^{10}+7765026192 a^{11}-552484058 a^{12} \\
& +6172320 a^{13}-4940 a^{14}-264 a^{15}+ \\
& +a^{16}-29375637080868126720000 c-33822953521504663633920 a c \\
& -11534741688339332628480 a^{2} c- \\
& -1247318164867203416064 a^{3} c+22820178739934550016 a^{4} c \\
& +7574669592594306048 a^{5} c- \\
& -84914980281410048 a^{6} c-17316950021682432 a^{7} c+433392396752128 a^{8} c \\
& +9606215587584 a^{9} c-
\end{aligned}
$$




$$
\begin{gathered}
-460516397824 a^{10} c+3803095296 a^{11} c+52672256 a^{12} c-1005312 a^{13} c+4352 a^{14} c \\
+26669053078361541181440 c^{2}+ \\
+22655654678828527386624 a c^{2}+5628843122258229460992 a^{2} c^{2} \\
+364720598945819922432 a^{3} c^{2}- \\
-22957429020158350336 a^{4} c^{2}-1849106009143236096 a^{5} c^{2} \\
+73265900144129792 a^{6} c^{2}+2327692620840576 a^{7} c^{2}-
\end{gathered}
$$$$
-122805604797568 a^{8} c^{2}+434231597184 a^{9} c^{2}+46423237504 a^{10} c^{2}-727106688 a^{11} c^{2}
$$$$
+1558144 a^{12} c^{2}+29568 a^{13} c^{2}-
$$$$
-128 a^{14} c^{2}-13276554097840248324096 c^{3}-8527557701096075034624 a c^{3}
$$$$
-1531141541685441052672 a^{2} c^{3}-
$$$$
-46499966481831751680 a^{3} c^{3}+6834918833916766208 a^{4} c^{3}
$$$$
+178742102347607040 a^{5} c^{3}-
$$

$-15578800189494784 a^{6} c^{3}-14483139922944 a^{7} c^{3}+12676556057088 a^{8} c^{3}$$$
-181619665920 a^{9} c^{3}-1088844288 a^{10} c^{3}+
$$$$
+36191232 a^{11} c^{3}-182784 a^{12} c^{3}+4183912176750576009216 c^{4}
$$$$
+2048627772744257667072 a c^{4}+
$$$$
+259599284321332817920 a^{2} c^{4}+476692959245589504 a^{3} c^{4}
$$$$
-1042172568281602560 a^{4} c^{4}+591530393836800 a^{5} c^{4}+
$$$$
+1548005345752448 a^{6} c^{4}-21488964279552 a^{7} c^{4}-529593246336 a^{8} c^{4}
$$$$
+12925059840 a^{9} c^{4}-46134144 a^{10} c^{4}-
$$$$
-532224 a^{11} c^{4}+2688 a^{12} c^{4}-901671972868968153088 c^{5}
$$$$
-336106259258033307648 a c^{5}-
$$

$-28768633067902959616 a^{2} c^{5}+678076651803893760 a^{3} c^{5}+92218372409180160 a^{4} c^{5}$ $-1692557156505600 a^{5} c^{5}-$

$-77849036998656 a^{6} c^{5}+1993171783680 a^{7} c^{5}+2814873600 a^{8} c^{5}-361912320 a^{9} c^{5}$

$$
+2193408 a^{10} c^{5}+
$$$$
+139192132696440569856 c^{6}+39163709061769003008 a c^{6}
$$$$
+2092243353303842816 a^{2} c^{6}-
$$

$-104389799581224960 a^{3} c^{6}-4779754566062080 a^{4} c^{6}+165520520782848 a^{5} c^{6}$

$+1574992505856 a^{6} c^{6}-$

$-79698769920 a^{7} c^{6}+428144640 a^{8} c^{6}+3548160 a^{9} c^{6}-21504 a^{1} 0 c^{6}$

$-15842385815912054784 c^{7}-$ 
$-7962071040000 a^{5} c^{7}+22036439040 a^{6} c^{7}+1516584960 a^{7} c^{7}-11489280 a^{8} c^{7}$ $+1352753717551824896 c^{8}+$

$+205696018035695616 a c^{8}+1846128096868352 a^{2} c^{8}-407844026320896 a^{3} c^{8}$ $+81687783936 a^{4} c^{8}+$

$+213236305920 a^{5} c^{8}-1724574720 a^{6} c^{8}-11151360 a^{7} c^{8}+84480 a^{8} c^{8}$ $-87414807455596544 c^{9}-$

$-9359636030029824 a c^{9}+52940629147648 a^{2} c^{9}+12879850536960 a^{3} c^{9}$ $-111737077760 a^{4} c^{9}-3033169920 a^{5} c^{9}+$

$+30638080 a^{6} c^{9}+4279210143121408 c^{10}+308423755825152 a c^{10}$

$-5039751135232 a^{2} c^{10}-254518640640 a^{3} c^{10}+$

$+3365683200 a^{4} c^{10}+17842176 a^{5} c^{10}-180224 a^{6} c^{10}-157590197633024 c^{11}$

$-7158795730944 a c^{11}+$

$+169615753216 a^{2} c^{11}+2867724288 a^{3} c^{11}-43450368 a^{4} c^{11}+4293100830720 c^{12}$ $+110927511552 a c^{12}-$

$-3129491456 a^{2} c^{12}-14057472 a^{3} c^{12}+212992 a^{4} c^{12}-83852525568 c^{13}$

$-1029439488 a c^{13}+31195136 a^{2} c^{13}+$

$\left.+1109917696 c^{14}+4325376 a c^{14}-131072 a^{2} c^{14}-8912896 c^{15}+32768 c^{16}\right)$

$+\frac{1}{\Gamma\left(\frac{c-a}{2}\right) \Gamma\left(\frac{c+a-33}{2}\right)}\left(-65536 c^{15}+16711680 c^{14}+229376 a^{2} c^{13}-7569408 a c^{13}\right.$

$-1944649728 c^{13}-$

$-50692096 a^{2} c^{12}+1672839168 a c^{12}+136767275008 c^{12}-319488 a^{4} c^{11}$

$+21086208 a^{3} c^{11}+4700200960 a^{2} c^{11}-$

$-166588071936 a c^{11}-6490170392576 c^{11}+59744256 a^{4} c^{10}-3943120896 a^{3} c^{10}$

$-234336886784 a^{2} c^{10}+$

$+9880146591744 a c^{10}+219687595212800 c^{10}+225280 a^{6} c^{9}-22302720 a^{5} c^{9}$ $-4212961280 a^{4} c^{9}+$

$+318534881280 a^{3} c^{9}+6385782734848 a^{2} c^{9}-388581283774464 a c^{9}$

$-5467350755508224 c^{9}-34467840 a^{6} c^{8}+$

$+3412316160 a^{5} c^{8}+126600376320 a^{4} c^{8}-14548978667520 a^{3} c^{8}-63061551218688 a^{2} c^{8}$ $+$ 
$+10677406306811904 a c^{8}+101605453158088704 c^{8}-84480 a^{8} c^{7}+11151360 a^{7} c^{7}$ $+1727278080 a^{6} c^{7}-$

$-213503938560 a^{5} c^{7}-132194118144 a^{4} c^{7}+411663197620224 a^{3} c^{7}$ $-1770221953955840 a^{2} c^{7}-$

$-210333358052302848 a c^{7}-1417367362882437120 c^{7}+10053120 a^{8} c^{6}$ $-1327011840 a^{7} c^{6}-19603584000 a^{6} c^{6}+$

$+6998660444160 a^{5} c^{6}-108366929459712 a^{4} c^{6}-7386371104831488 a^{3} c^{6}$ $+81588957922920448 a^{2} c^{6}+$

$+2998385401531441152 a c^{6}+14789061893062983680 c^{6}+16128 a^{10} c^{5}-2661120 a^{9} c^{5}$ $-321699840 a^{8} c^{5}+$

$+59852136960 a^{7} c^{5}-1169166679296 a^{6} c^{5}-125633606757120 a^{5} c^{5}$

$+3584138069952000 a^{4} c^{5}+$

$+81155276599034880 a^{3} c^{5}-1581946349642745856 a^{2} c^{5}-30822380253791182848 a c^{5}$

$-113998595959458562048 c^{5}-1370880 a^{10} c^{4}+226195200 a^{9} c^{4}-1709030400 a^{8} c^{4}$ $-1252367424000 a^{7} c^{4}+$

$+48558756153600 a^{6} c^{4}+1092730056042240 a^{5} c^{4}-58182434494609920 a^{4} c^{4}$ $-460255718632596480 a^{3} c^{4}+$

$+18390328035742767104 a^{2} c^{4}+224712599605813321728 a c^{4}$ $+634238712296638578688 c^{4}-1344 a^{12} c^{3}+$

$+266112 a^{11} c^{3}+23120832 a^{10} c^{3}-6471400320 a^{9} c^{3}+263725670208 a^{8} c^{3}$ $+10943807124096 a^{7} c^{3}-$

$-777928079414464 a^{6} c^{3}-711059420649600 a^{5} c^{3}+533035048250363648 a^{4} c^{3}$ $+25486745680704000 a^{3} c^{3}-$

$-135043097758270087168 a^{2} c^{3}-1123667660559841959936 a c^{3}$ $-2449470002402175418368 c^{3}+68544 a^{12} c^{2}-$

$-13571712 a^{11} c^{2}+405574848 a^{10} c^{2}+68559765120 a^{9} c^{2}-4757196954048 a^{8} c^{2}$ $+2935731705984 a^{7} c^{2}+$

$+5939303383785024 a^{6} c^{2}-64891677848976000 a^{5} c^{2}-2678695653148796160 a^{4} c^{2}+$ $+16568080735699671552 a^{3} c^{2}+610385093977265700864 a^{2} c^{2}$ $+3626708216318306279424 a c^{2}+$

$+6147778466426910474240 c^{2}+32 a^{14} c-7392 a^{13} c-390880 a^{12} c+182042784 a^{11} c$ $-11582726176 a^{10} c-$ 


$$
\begin{gathered}
-115023090336 a^{9} c+30965876629600 a^{8} c-571118887841568 a^{7} c \\
-19091657228488064 a^{6} c+ \\
+461856383439694848 a^{5} c+6264028269209728000 a^{4} c-91339795704196148736 a^{3} c \\
-\quad 8864971566659828121600 c-544 a^{14}+ \\
-1538581445063915139072 a^{2} c-6717806419589716746240 a c \\
+125664 a^{13}-6561184 a^{12}-479910816 a^{11}+57700381088 a^{10}-1178029251168 a^{9} \\
-55758969524192 a^{8}+ \\
+2166179809154592 a^{7}+12571450930103296 a^{6}-968963896585149696 a^{5} \\
-3718430128264001024 a^{4}+ \\
+161641469374376538624 a^{3}+1636843212624447221760 a^{2} \\
+5336242598307820953600 a+)
\end{gathered}
$$

\section{EVALUATION OF THE MAIN FORMULAE}

Involving the contiguous relation (8) and the formula of Salahuddin et al (33), the result (34) can be established and on the same way result(35) can be established.

\section{References}

1. Abramowitz, Milton., A and Stegun, Irene; Handbook of Mathematical Functions with Formulas, Graphs, and Mathematical Tables. National Bureau of Standards, 1970.

2. Husain, Intazar and Salahuddin; Two Ridiculous Summation Formulae arising from the summation formulae of Salahuddin et al, International Research Journal of Engineering and Technology,3(2016),110-120.

3. Prudnikov, A.P., Brychkov, Yu. A. and Marichev, O.I.; Integral and Series Vol 3: More Special Functions, Nauka, Moscow,2003.

4. Salahuddin, Khola, R. K.; New hypergeometric summation formulae arising from the summation formulae of Prudnikov, South Asian Journal of Mathematics,4(2014),192-196.

5. Salahuddin, Khola, R. K.; New Prospective of Hypergeometric Summation, Sohag Journal of Mathematics,3(2016),1-7.

6. Salahuddin, Khola, R. K.; Two Curious Summation Formulae in the Monograph of Salahuddin Et Al, Global Journal of Science Frontier Research: F,16(2016),67-73.

7. Salahuddin, Pandit, U. K. and Chaudhary, M. P.; Two Incredible Summation Formula Involving Computational Technique, Global Journal of Science Frontier Research: $F, 15(2015), 29-35$.

8. Salahuddin, Chaudhary, M. P.; Two Wondrous Summation Formulae, Pacific Journal of Applied Mathematics,8(2016),103-113.

9. Salahuddin; Two new magnificent summation formulae, South Asian Journal of Mathematics,6(2016),152-168. 